\title{
CLASH-VLT: INSIGHTS ON THE MASS SUBSTRUCTURES IN THE FRONTIER FIELDS CLUSTER MACS J0416.1-2403 THROUGH ACCURATE STRONG LENS MODELING*
}

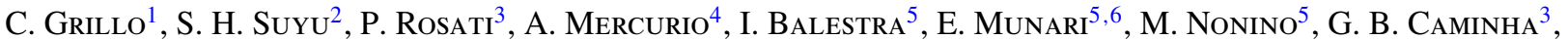 \\ M. Lombardi ${ }^{7}$, G. De Lucia ${ }^{5}$, S. Borgani ${ }^{5,6}$, R. Gobat ${ }^{8}$, A. Biviano ${ }^{5}$, M. Girardi ${ }^{5,6}$, K. Umetsu $^{2}$, D. Coe ${ }^{9}$, \\ A. M. Koekemoer ${ }^{9}$, M. Postman ${ }^{9}$, A. Zitrin ${ }^{10,16}$, A. Halkola, T. Broadhurst ${ }^{11}$, B. Sartoris ${ }^{6}$, \\ V. Presotto ${ }^{6}$, M. Annunziatella ${ }^{5,6}$, C. Maier $^{12}$, A. Fritz $^{13}$, E. Vanzella ${ }^{14}$, And B. Frye ${ }^{15}$ \\ ${ }^{1}$ Dark Cosmology Centre, Niels Bohr Institute, University of Copenhagen, Juliane Maries Vej 30, \\ DK-2100 Copenhagen, Denmark; grillo@ dark-cosmology.dk \\ ${ }^{2}$ Institute of Astronomy and Astrophysics, Academia Sinica, P.O. Box 23-141, Taipei 10617, Taiwan \\ ${ }^{3}$ Dipartimento di Fisica e Scienze della Terra, Università degli Studi di Ferrara, Via Saragat 1, I-44122 Ferrara, Italy \\ ${ }^{4}$ INAF - Osservatorio Astronomico di Capodimonte, Via Moiariello 16, I-80131 Napoli, Italy \\ ${ }^{5}$ INAF - Osservatorio Astronomico di Trieste, via G. B. Tiepolo 11, I-34143, Trieste, Italy \\ ${ }^{6}$ Dipartimento di Fisica, Università degli Studi di Trieste, via G. B. Tiepolo 11, I-34143 Trieste, Italy \\ ${ }^{7}$ Dipartimento di Fisica, Università degli Studi di Milano, via Celoria 16, I-20133 Milano, Italy \\ ${ }^{8}$ Laboratoire AIM-Paris-Saclay, CEA/DSM-CNRS-Universitè Paris Diderot, Irfu/Service d'Astrophysique, \\ CEA Saclay, Orme des Merisiers, F-91191 Gif sur Yvette, France \\ ${ }^{9}$ Space Telescope Science Institute, 3700 San Martin Drive, Baltimore, MD 21208, USA \\ ${ }^{10}$ Cahill Center for Astronomy and Astrophysics, California Institute of Technology, MS 249-17, Pasadena, CA 91125, USA \\ ${ }^{11}$ Ikerbasque, Basque Foundation for Science, Alameda Urquijo, 36-5 Plaza Bizkaia, E-48011, Bilbao, Spain \\ ${ }^{12}$ University of Vienna, Department of Astrophysics, Türkenschanzstr. 17, A-1180, Wien, Austria \\ ${ }^{13}$ INAF - Istituto di Astrofisica Spaziale e Fisica cosmica (IASF) Milano, via Bassini 15, I-20133 Milano, Italy \\ ${ }^{14}$ INAF - Osservatorio Astronomico di Bologna, Via Ranzani 1, I- 40127 Bologna, Italy \\ ${ }^{15}$ Department of Astronomy/Steward Observatory, University of Arizona, 933 North Cherry Avenue, Tucson, AZ 85721, USA \\ Received 2014 July 28; accepted 2014 December 9; published 2015 February 6
}

\begin{abstract}
We present a detailed mass reconstruction and a novel study on the substructure properties in the core of the Cluster Lensing And Supernova survey with Hubble (CLASH) and Frontier Fields galaxy cluster MACS J0416.1-2403. We show and employ our extensive spectroscopic data set taken with the VIsible Multi-Object Spectrograph instrument as part of our CLASH-VLT program, to confirm spectroscopically 10 strong lensing systems and to select a sample of 175 plausible cluster members to a limiting stellar mass of $\log \left(M_{*} / M_{\odot}\right) \simeq 8.6$. We reproduce the measured positions of a set of 30 multiple images with a remarkable median offset of only 0.3 by means of a comprehensive strong lensing model comprised of two cluster dark-matter halos, represented by cored elliptical pseudo-isothermal mass distributions, and the cluster member components, parameterized with dual pseudo-isothermal total mass profiles. The latter have total mass-to-light ratios increasing with the galaxy HST/WFC3 near-IR (F160W) luminosities. The measurement of the total enclosed mass within the Einstein radius is accurate to $\sim 5 \%$, including the systematic uncertainties estimated from six distinct mass models. We emphasize that the use of multiple-image systems with spectroscopic redshifts and knowledge of cluster membership based on extensive spectroscopic information is key to constructing robust high-resolution mass maps. We also produce magnification maps over the central area that is covered with HST observations. We investigate the galaxy contribution, both in terms of total and stellar mass, to the total mass budget of the cluster. When compared with the outcomes of cosmological $N$-body simulations, our results point to a lack of massive subhalos in the inner regions of simulated clusters with total masses similar to that of MACS J0416.1-2403. Our findings of the location and shape of the cluster dark-matter halo density profiles and on the cluster substructures provide intriguing tests of the assumed collisionless, cold nature of dark matter and of the role played by baryons in the process of structure formation.
\end{abstract}

Key words: dark matter - galaxies: clusters: general - galaxies: clusters: individual (MACS J0416.1-2403) gravitational lensing: strong

\section{INTRODUCTION}

The currently accepted cold dark matter dominated model with the cosmological constant $(\Lambda \mathrm{CDM})$ predicts that structures in our Universe assemble hierarchically, with more massive systems forming later through accretion and mergers of smaller, self-bound dark-matter halos (e.g., Tormen 1997; Moore et al. 1999; Klypin et al. 1999; Springel et al. 2001). In $N$-body cosmological simulations, dark matter halos of all masses

\footnotetext{
* This work is based in large part on data collected at ESO VLT (prog. ID 186.A-0798) and NASA $H S T$.

${ }^{16}$ Hubble Fellow.
}

converge to a roughly "universal" and cuspy density profile that steepens with radius, the so-called Navarro-Frenk-White profile (NFW profile; Navarro et al. 1996, 1997). Moreover, the degree of central concentration of a halo depends on its formation epoch and hence on its total mass (e.g., Wechsler et al. 2002; Zhao et al. 2003). Within this scenario, early virialized objects are compact when they get accreted into a larger halo. Such objects are usually referred to as subhalos or substructures of their host and, as they orbit within the host potential well, they are strongly affected by tidal forces and dynamical friction, causing mass, angular momentum, and energy loss (e.g., Ghigna et al. 1998; Tormen et al. 1998; De Lucia et al. 2004; Gao et al. 2004). In the $\Lambda$ CDM framework, more massive halos are 
predicted to have a larger fraction of mass in subhalos than lower mass halos because in the former there has been less time for tidal destruction to take place (e.g., Gao et al. 2004; Contini et al. 2012). On galaxy cluster scales, observational tests of these predictions have been attempted in some previous works (e.g., Natarajan et al. 2007, 2009), but highly accurate analyses are becoming possible only now, thanks to the substantially improved quality of the available photometric and spectroscopic data. From an observational point of view, more investigations are still required to fully answer key questions on the formation and evolution of subhalos. How much mass of subhalos is stripped as they fall into the host potential? How many subhalos survive as bound objects? What are the spatial and mass distributions of the subhalos?

Significant progress (Biviano et al. 2013; Lemze et al. 2013; Umetsu et al. 2014; Merten et al. 2014; Meneghetti et al. 2014; Donahue et al. 2014) in the fields of galaxy cluster formation and evolution has lately been made thanks to the data collected within the Hubble Space Telescope (HST) MultiCycle Treasury program Cluster Lensing And Supernova survey with Hubble (CLASH; P.I.: M. Postman; Postman et al. 2012), often complemented with the spectroscopic campaign carried out with the Very Large Telescope (VLT; the CLASH-VLT Large Programme; P.I.: P. Rosati; P. Rosati et al., in preparation). Recent new results from systematic in-depth studies of lensing clusters with $H S T$ have led to the conception of the Hubble Frontier Fields (HFF; P.I.: J. Lotz) that will target, using Director Discretionary Time, up to six massive galaxy clusters, for a total of 140 HST orbits on each cluster, in 7 broadband filters, achieving in all of them unprecedented depth of $\approx 29$ mag $(A B)$. Not only will this program detect the highest redshift galaxies and characterize for the first time this sample of star-forming galaxies in a statistically meaningful way, the HFF data will provide a great opportunity to study the structure of the dark matter halos hosting these clusters.

In this paper, we focus on the HFF cluster MACS J0416.1-2403 (hereafter MACS 0416) that was first discovered in the X-rays by Mann \& Ebeling (2012) as part of the Massive Cluster Survey (MACS). MACS 0416 is an elongated cluster undergoing a merger, and such an elongated geometry makes MACS 0416 an efficient gravitational lens for highly magnifying background sources and forming multiple images of each background source (the regime of "strong" lensing). Given its high magnifications and the upcoming HFF infrared observations, there has been several recent studies of MACS 0416. In particular, Zitrin et al. (2013) has identified 70 multiple images and candidates that are associated with 23 background sources, confirming the enhanced lensing efficiency of MACS 0416 relative to other clusters. Jauzac et al. (2014) further identified 51 strongly lensed background sources, yielding 194 multiple images of lensed background sources. Jauzac et al. (2015) further complement the strong lensing data with weak lensing and X-ray observations to study the dynamics of MACS 0416. Using a free-form mass modeling approach, Diego et al. (2014) found that the mass distribution in MACS 0416 overall traces its light distribution. Johnson et al. (2014) and Richard et al. (2014) have also modeled MACS 0416 as part of the HFF sample, and provided the mass and magnifications maps of the clusters.

Building upon and extending these previous studies, we perform a thorough strong lensing analysis of MACS 0416 with the following new ingredients: (1) a large number of spectroscopic redshifts of strongly lensed background sources obtained through our CLASH-VLT program, (2) a robust approach
Table 1

Photometric and Spectroscopic Properties of the Two Brightest Cluster Galaxies G1 and G2

\begin{tabular}{lcccccc}
\hline \hline ID & $\begin{array}{c}\text { R.A. } \\
(\mathrm{J} 2000)\end{array}$ & $\begin{array}{c}\text { Decl. } \\
(\mathrm{J} 2000)\end{array}$ & $\begin{array}{c}x^{\mathrm{a}} \\
\left({ }^{\prime \prime}\right)\end{array}$ & $\begin{array}{c}y^{\mathrm{a}} \\
\left(^{\prime \prime}\right)\end{array}$ & $z_{\text {sp }}$ & $\begin{array}{c}\text { F160W } \\
(\mathrm{mag})\end{array}$ \\
\hline G1 & $04: 16: 09.154$ & $-24: 04: 02.90$ & $\equiv 0.000$ & $\equiv 0.000$ & 0.400 & 17.02 \\
G2 & $04: 16: 07.671$ & $-24: 04: 38.75$ & 20.310 & -35.846 & 0.396 & 17.24 \\
\hline
\end{tabular}

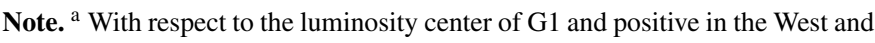
North directions.

of selecting cluster galaxies based on multi-color information calibrated on 113 spectroscopic members in the HST field of view (FoV), and (3) a detailed mass model that tests various methodological assumptions with our best model reproducing the observed multiple-image positions substantially better than all previous studies. Using our mass model, we compare the distribution of the cluster galaxies with those of the cluster subhalos from $N$-body simulations to probe with unexampled accuracy the substructure properties of a galaxy cluster.

The outline of the paper is as follows. In Section 2, we describe the imaging and spectroscopic observations of MACS 0416. We detail our strong lens modeling of the cluster in Section 3, including the selection of background source galaxies and cluster galaxies. Our resulting mass model is then compared to those published in the literature in Section 4. We compare in Section 5 the mass distribution of the cluster galaxies of MACS 0416 with those of the cluster subhalos of analog clusters from $\mathrm{N}$-body simulations, before presenting conclusions in Section 6.

Throughout this paper, we assume a flat $\Lambda \mathrm{CDM}$ cosmology with $H_{0}=70 \mathrm{~km} \mathrm{~s}^{-1} \mathrm{Mpc}^{-1}, \Omega_{\Lambda}=1-\Omega_{\mathrm{m}}=0.7$. In this cosmology, $1^{\prime \prime}$ corresponds to $5.34 \mathrm{kpc}$ at the lens redshift of $z_{\text {lens }}=0.396$. All magnitudes are given in the AB system. Parameter constraints are given as the median values with uncertainties given by the 16th and 84th percentiles (corresponding to $68 \%$ confidence levels (CLs)) unless otherwise stated.

\section{DATA}

\subsection{HST Imaging}

Being a target of the CLASH program, MACS 0416 was observed in HST Cycle 19, between 2012 July 24 and September 27 , in 16 broadband filters, from the UV to the near-IR, to a total depth of 20 orbits (see Postman et al. 2012). The images were processed for debias, flats, superflats, and darks using standard techniques, and then co-aligned and combined using drizzle algorithms to pixel scales of 0.030 and 0'.065 (for details, see Koekemoer et al. 2007, 2011).

A color image of the inner regions of the galaxy cluster, obtained through a combination of the HST/ACS and WFC3 filters, is shown in Figure 1. There, we have marked the two brightest galaxies of the cluster, G1 and G2 (see also Table 1), and the multiple image systems studied in this paper (see Section 3.1).

\subsection{VLT Spectroscopy}

MACS 0416 was first observed between 2012 December and 2013 February, as part of the ESO Large Programme 186.A-0798 “Dark Matter Mass Distributions of Hubble Treasury Clusters and the Foundations of $\Lambda$ CDM Structure Formation Models" (CLASH-VLT) using the VIsible Multi-Object Spectrograph (VIMOS; Le Fèvre et al. 2003) at the ESO VLT. 


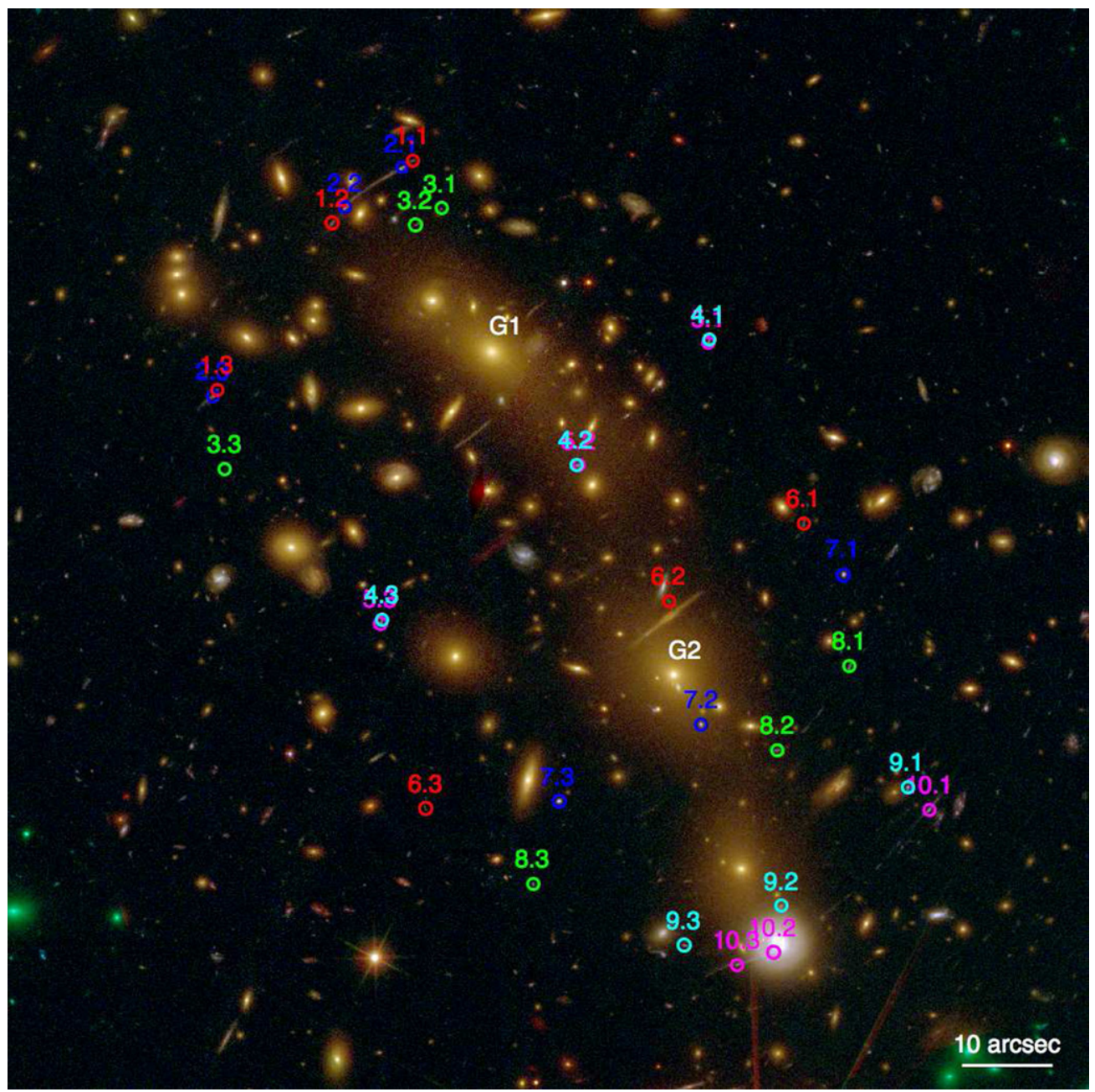

Figure 1. $2^{\prime} \times 2^{\prime}$ color-composite image of MACS J0416.1-2403 obtained by combining the 16 filters of HST/ACS and WFC3. North is top and east is left. The thirty multiple images modeled in this paper and the two brightest cluster galaxies, G1 and G2, are labeled. More information about these objects is provided in Tables 1 and 3 and Figure 2.

The VIMOS data were acquired using eight pointings with one quadrant always locked on the cluster core, thus allowing longer exposures on the arcs. The exposure time for each pointing was 60 minutes, except for the last two pointings that had shorter exposure times ( 30 minutes) because they targeted cluster member galaxies. Therefore, the final integration times for arcs and other background galaxies varied between 30 minutes and $4 \mathrm{hr}$. A $\log$ of our VIMOS observations is presented in Table 2. We used the LR-blue grism, with a spectral resolution of approximately $28 \AA$ with $1^{\prime \prime}$-slits and a wavelength coverage of 3700-6700 А.

We assign a quality flag (QF; indicated as Quality in Figures 2 and 3) to each redshift, which indicates the reliability of a redshift measurement. We define four redshift quality classes: $\operatorname{SECURE}(\mathrm{QF}=3)$, LIKELY $(\mathrm{QF}=2)$, INSECURE $(\mathrm{QF}=1)$, and BASED ON A SINGLE EMISSION-LINE $(\mathrm{QF}=9)$. To assess the reliability of these four quality classes we compared pairs of duplicate observations having at least one secure measurement. In this way, we could quantify the reliability of each quality class as follows: redshifts with $\mathrm{QF}=3$ are correct with a probability of $>99.99 \%, \mathrm{QF}=9$ with $\sim 92 \%$ probability, $\mathrm{QF}=2$ with $\sim 75 \%$ probability, and $\mathrm{QF}=1$ with $<40 \%$ probability. In this paper we will only consider redshifts with $\mathrm{QF}=3,2$, or 9 . To date, we have 4160 reliable redshifts over a field $\sim 25$ arcmin across, over 800 of which are cluster members. Full details on
Table 2

Log of VIMOS Observations of MACS 0416, Taken as Part of our CLASH-VLT Spectroscopic Campaign

\begin{tabular}{lcc}
\hline \hline $\begin{array}{l}\text { OBS ID } \\
(1)\end{array}$ & $\begin{array}{c}\text { Date } \\
(2)\end{array}$ & $\begin{array}{c}\text { Exp. Time (minute) } \\
(3)\end{array}$ \\
\hline \multicolumn{3}{c}{ LR-blue masks } \\
\hline 848955 & 2012 Dec & 60 \\
848957 & $2013 \mathrm{Jan}$ & 60 \\
848959 & $2012 \mathrm{Dec}$ & 60 \\
856270 & $2013 \mathrm{Feb}$ & 60 \\
916733 & 2013 Feb & 60 \\
916723 & 2013 Feb & 60 \\
915893 & 2013 Feb & 30 \\
915903 & $2013 \mathrm{Feb}$ & 30 \\
\hline
\end{tabular}

Notes. Columns list the following information: (1) VIMOS mask identification number, (2) date of the observations, and (3) exposure time.

the spectroscopic sample observations and data reduction will be given in I. Balestra et al. (in preparation).

In the spirit of the open-data access of the HFF initiative, we had an early release (2013 July) of a redshift catalog of 118 sources in the HST FoV, including the redshifts of the multiple image systems presented in the next section. This information 

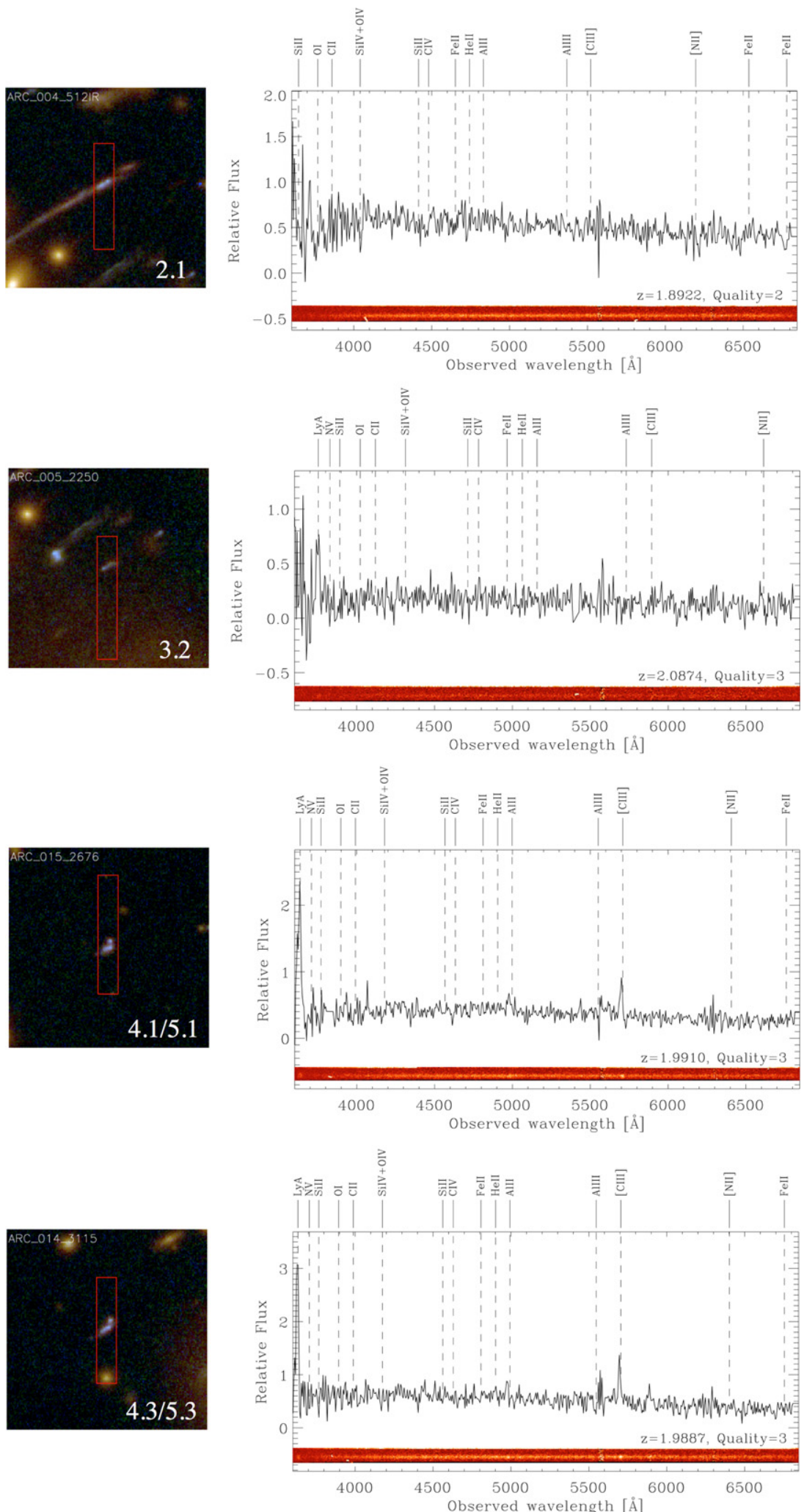

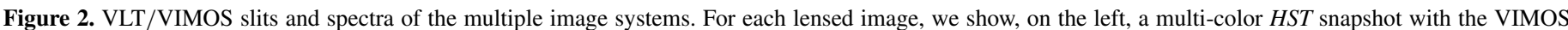

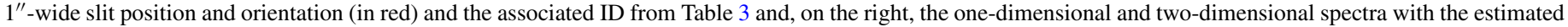

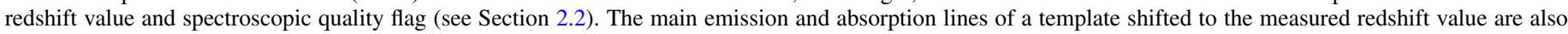
indicated.

has been used when building recent lensing models of MACS 0416 (see Section 4; Johnson et al. 2014; Richard et al. 2014; Jauzac et al. 2014, 2015; Diego et al. 2014). We note that the redshift of system 7 (see Table 3 ) has been revised to a more reliable value of 1.637 , thanks to spectroscopic data that became available only after our first release. As a result of our continuing spectroscopic campaign, which will be completed at the end of 2014, in this paper we use the current spectroscopic redshift 

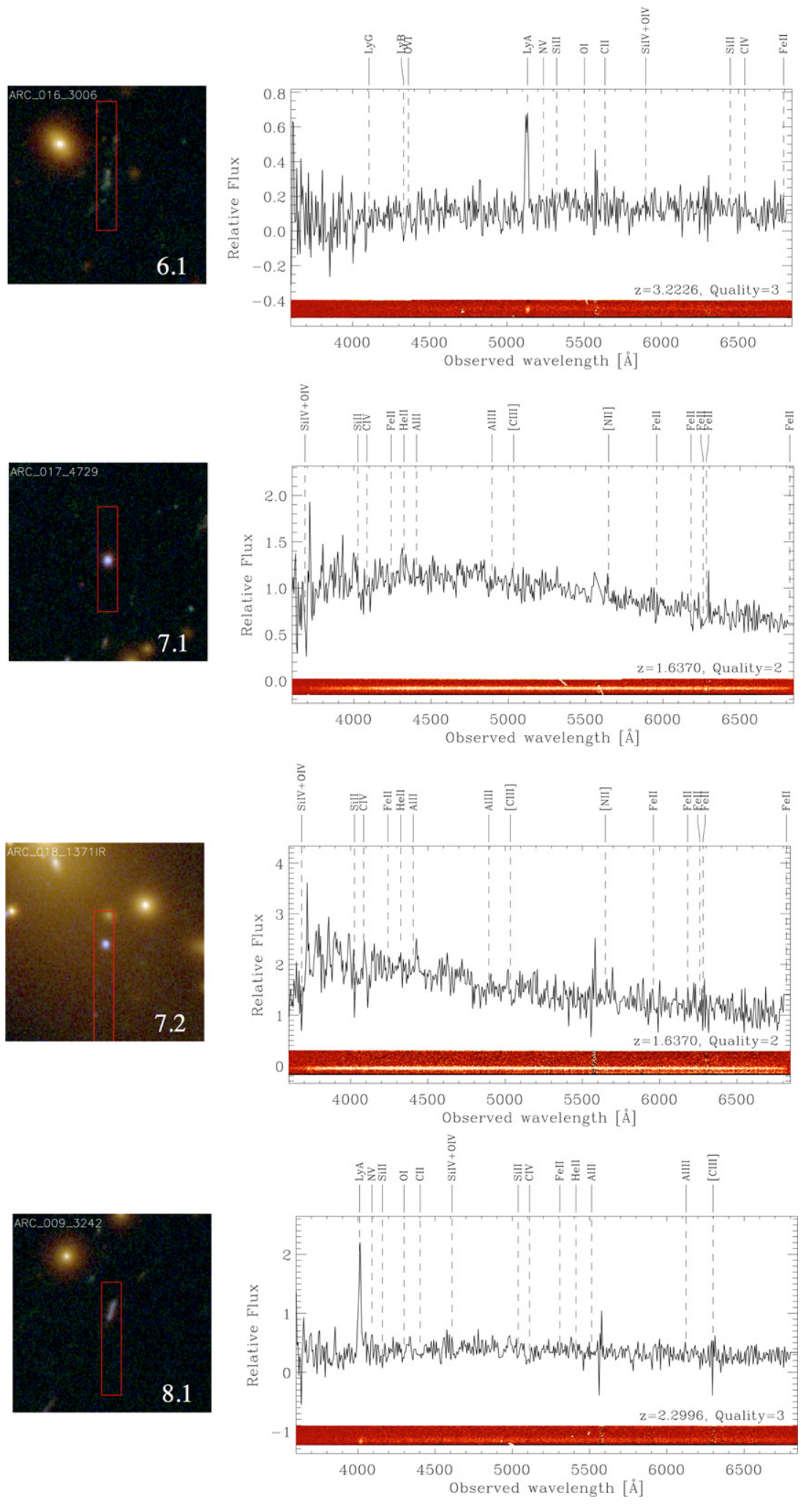

Figure 2. (Continued)

information for 215 galaxies in the HST FoV, 113 of which are cluster members (see Section 3.3.1).

\section{LENS MODELING}

We describe the mass modeling of MACS 0416 based on the strong lensing features. In Section 3.1, we identify the multiple image systems of strongly lensed background sources. In Section 3.2, we give an overview of the method and software used to model the lens mass distribution, with the decomposition of cluster members and extended dark-matter halos described in Section 3.3. We detail our collection of mass models of the galaxy cluster in Section 3.4, and present the resulting total and luminous mass distribution of MACS 0416 in Section 3.5. 

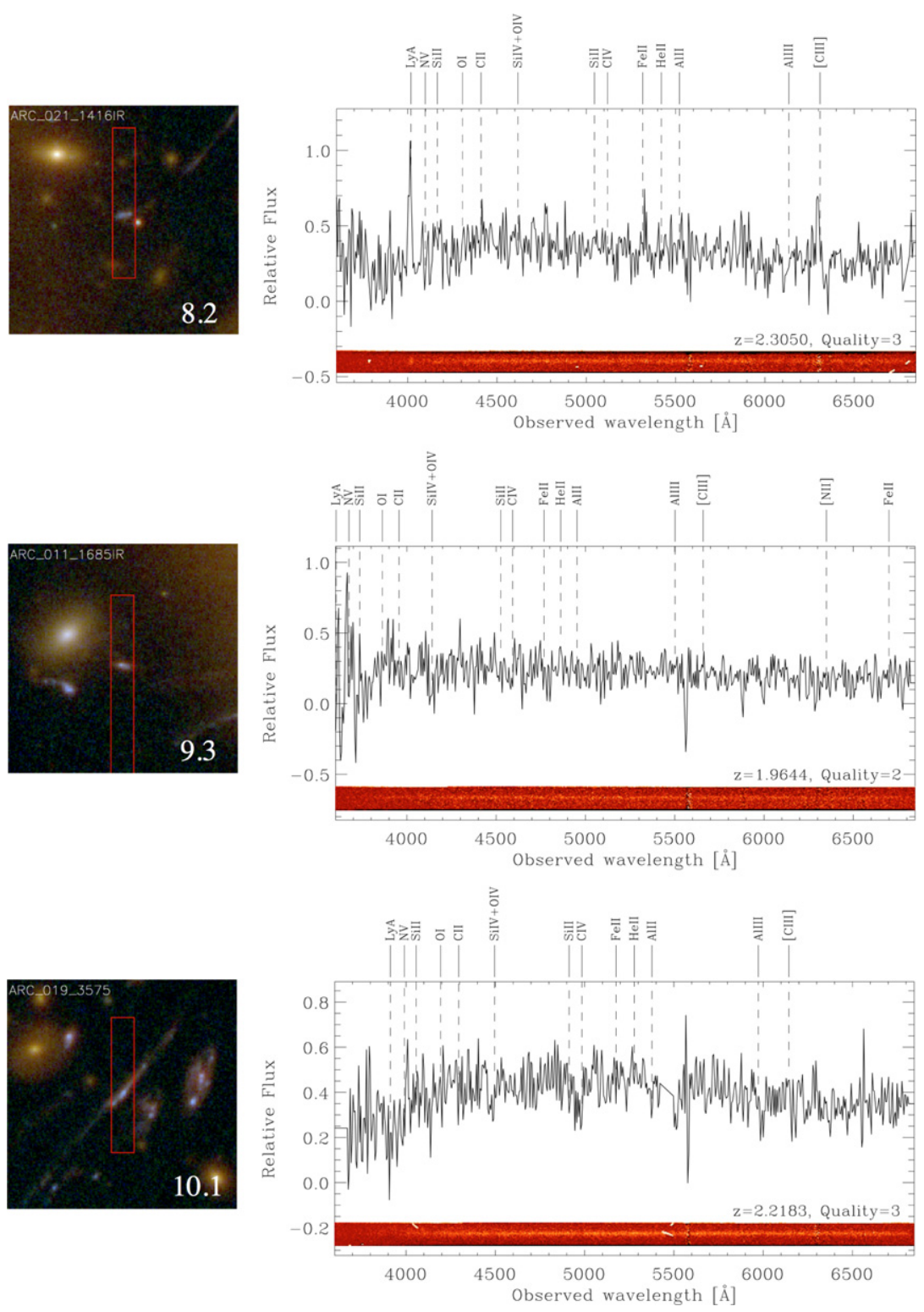

Figure 2. (Continued)

\subsection{Multiple Image Systems}

The physical observables that we want to reconstruct are the positions of ten multiple image systems, each of which is composed of three images associated to one background source. We choose our multiple image systems among the reliable or candidate systems selected by Zitrin et al. (2013) and now spectroscopically confirmed by our VLT/VIMOS observations. Every system has at least one multiple image with a spectroscopic redshift value classified as either SECURE (i.e., Quality $=3$ in Figure 2) or LIKELY (i.e., Quality $=2$ in Figure 2), according to the criteria defined in Section 2.2. If a system has spectroscopic observations for two images, both of which with SECURE estimates, we adopt for that system the average of the SECURE redshift values. If a system has spectroscopic observations for two images, one with a SECURE and the other with a LIKELY estimate, we adopt for that system the SECURE redshift value. In Figure 1, we show that the multiple image systems cover a relatively large area of the cluster central region and are distributed in a fairly uniform way around the two brightest cluster members G1 and G2. We remark that all sources are rather compact and well approximated by point-like objects. Nonetheless, to exploit better the information contained in the surface brightness distribution of two sources, we split each of these sources into two systems (see in Figure 1, systems 1 and 2 and systems 4 and 5).

The observed angular positions, $x$ and $y$ (measured with respect to the luminosity center of the galaxy G1 and positive in the West and North directions), and spectroscopic redshifts, $z_{\mathrm{sp}}$, of the thirty multiple images are listed in Table 3 . The positional uncertainty for each image, $\delta_{x, y}$, is one pixel of the chosen HST images (i.e., 0.'065). In Figure 2, we show HST color-composite snapshots, with the VIMOS 1"-wide slits marked, and the reduced two-dimensional and one-dimensional spectra with the estimated redshift values of the targeted objects. We notice that the background lensed sources span a relatively large redshift range extending from 1.637 to 3.223 . In total, the multiple images provide sixty observables to be reproduced by a strong lensing model.

In Table 4 and Figure 3, we present three additional background sources with reliable high-redshift measurements. They are located outside the strong lensing region, where multiple 

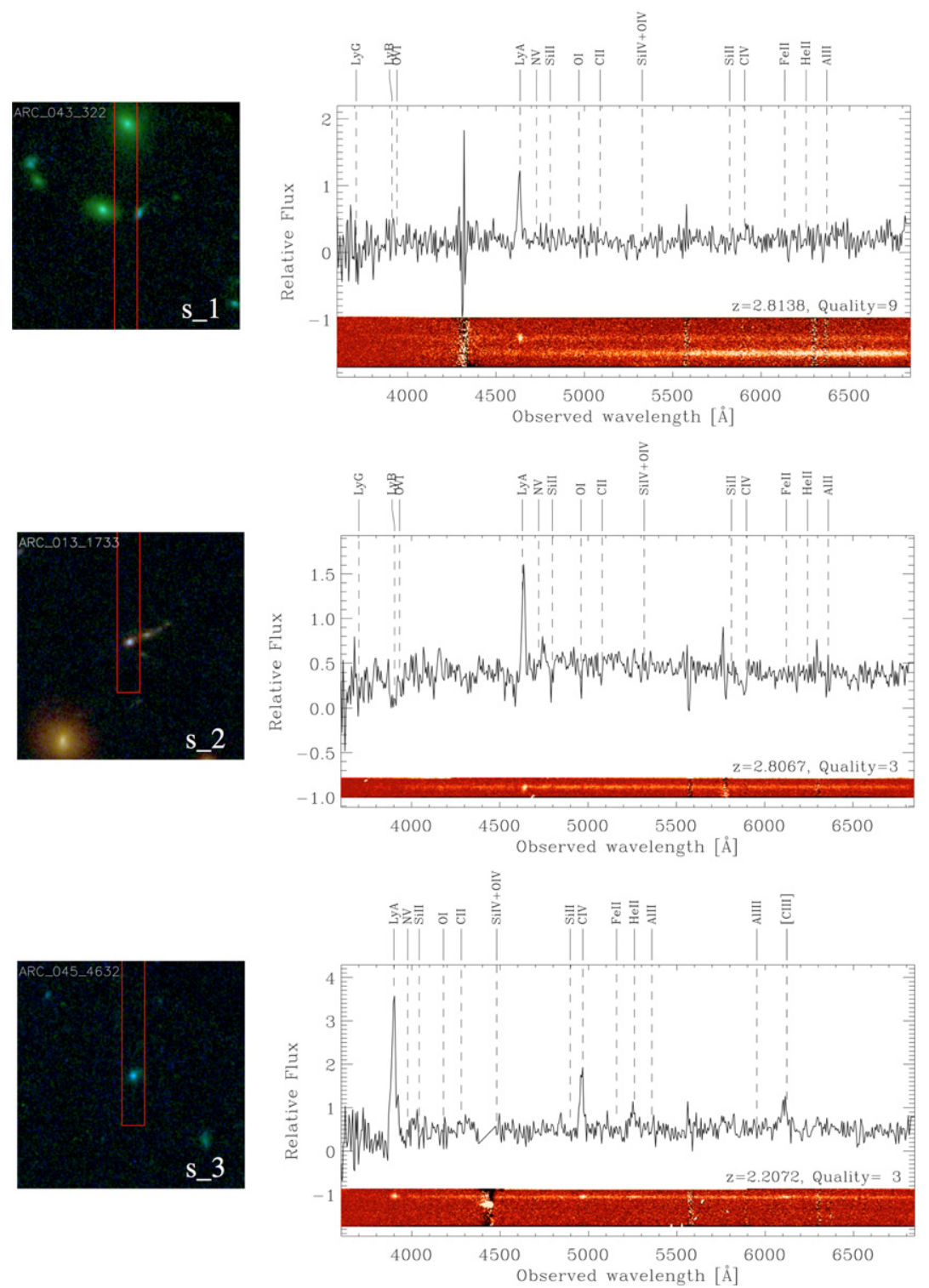

Figure 3. VLT/VIMOS slits and spectra of the images that are magnified but not multiply imaged. For each magnified image, we show, on the left, a multi-color $H S T$ snapshot with the VIMOS 1"-wide slit position and orientation (in red) and the associated ID from Table 4 and, on the right, the one-dimensional and two-dimensional spectra with the estimated redshift value and spectroscopic quality flag (see Section 2.2). The main emission and absorption lines of a template shifted to the measured redshift value are also indicated.

images of a source are created. Therefore, these objects are distorted and magnified, but not multiply imaged, by the cluster lensing effect.

\subsection{GLEE}

We model the mass distribution of MACS 0416 with GLEE, a software developed by A. Halkola and S. H. Suyu (Suyu \& Halkola 2010; Suyu et al. 2012). We use simply parameterized mass profiles to describe the cluster galaxies and dark matter halo, and we denote the lens parameters collectively as $\eta$. The image positions of the 10 multiple image systems in Table 3 are then used to constrain the parameters $\boldsymbol{\eta}$.

We use Bayesian analysis to infer the mass model parameters. In particular, we sample the posterior probability distribution function (PDF) of the lens mass parameters $\boldsymbol{\eta}$ given the data of observed image positions $\boldsymbol{d}_{\text {pos }}$,

$$
P\left(\boldsymbol{\eta} \mid \boldsymbol{d}_{\text {pos }}\right) \propto P\left(\boldsymbol{d}_{\text {pos }} \mid \boldsymbol{\eta}\right) P(\boldsymbol{\eta}) .
$$

The proportionality in the above equation follows from Bayes' Theorem, and the first term to the right of the proportionality is the likelihood whereas the second term is the prior. The likelihood of the lensing data is

$$
P\left(\boldsymbol{d}_{\text {pos }} \mid \boldsymbol{\eta}\right)=\frac{1}{Z_{\mathrm{pos}}} \exp \left[-\frac{1}{2} \sum_{j=1}^{N_{\mathrm{sys}}} \sum_{i=1}^{N_{\mathrm{m}}^{j}} \frac{\left|\boldsymbol{R}_{i, j}^{\mathrm{obs}}-\boldsymbol{R}_{i, j}^{\mathrm{pred}}(\boldsymbol{\eta})\right|^{2}}{\sigma_{i, j}^{2}}\right],
$$

where $N_{\text {sys }}$ is the number of multiply imaged systems $(=10$, as listed in Table 3), $N_{\mathrm{im}}^{j}$ is the number of multiple images in system $j, \boldsymbol{R}_{i, j}^{\text {obs }}=\left(x_{i, j}^{\text {obs }}, y_{i, j}^{\text {obs }}\right)$ is the observed image position, $\boldsymbol{R}_{i, j}^{\text {pred }}(\boldsymbol{\eta})$ is the predicted/modeled image position (given the lens parameters $\boldsymbol{\eta}), \sigma_{i, j}$ is the uncertainty in the observed image position, and $Z_{\mathrm{pos}}$ is the normalization given by

$$
Z_{\mathrm{pos}}=(2 \pi)^{N_{\mathrm{pos}}} \prod_{j=1}^{N_{\mathrm{sys}}} \prod_{i=1}^{N_{\mathrm{m}}^{j}} \sigma_{i, j}^{2}
$$


Table 3

Photometric and Spectroscopic Properties of the Multiple Image Systems

\begin{tabular}{|c|c|c|c|c|c|c|c|}
\hline ID & $\begin{array}{c}\text { R.A. } \\
\text { (J2000) }\end{array}$ & $\begin{array}{c}\text { Decl. } \\
(\mathrm{J} 2000)\end{array}$ & $\begin{array}{c}x^{\mathrm{a}} \\
\left({ }^{\prime \prime}\right)\end{array}$ & $\begin{array}{c}y^{\mathrm{a}} \\
\left({ }^{\prime \prime}\right)\end{array}$ & $z_{\mathrm{sp}}$ & $\begin{array}{c}\delta_{x, y} \\
\left({ }^{\prime \prime}\right)\end{array}$ & ID $Z 13^{b}$ \\
\hline 1.1 & 04:16:09.784 & $-24: 03: 41.76$ & -8.626 & 21.137 & 1.892 & 0.065 & 1.1 \\
\hline 1.2 & $04: 16: 10.435$ & $-24: 03: 48.69$ & -17.549 & 14.214 & 1.892 & 0.065 & 1.2 \\
\hline 1.3 & $04: 16: 11.365$ & $-24: 04: 07.21$ & -30.285 & -4.312 & 1.892 & 0.065 & 1.3 \\
\hline 2.1 & 04:16:09.871 & $-24: 03: 42.59$ & -9.823 & 20.308 & 1.892 & 0.065 & 2.1 \\
\hline 2.2 & $04: 16: 10.329$ & $-24: 03: 46.96$ & -16.101 & 15.937 & 1.892 & 0.065 & 2.2 \\
\hline 2.3 & 04:16:11.395 & $-24: 04: 07.86$ & -30.698 & -4.962 & 1.892 & 0.065 & 2.3 \\
\hline 3.1 & 04:16:09.549 & $-24: 03: 47.08$ & -5.419 & 15.819 & 2.087 & 0.065 & c7.1 \\
\hline 3.2 & 04:16:09.758 & $-24: 03: 48.90$ & -8.282 & 14.001 & 2.087 & 0.065 & c7.2 \\
\hline 3.3 & $04: 16: 11.304$ & $-24: 04: 15.94$ & -29.451 & -13.040 & 2.087 & 0.065 & c7.3 \\
\hline 4.1 & $04: 16: 07.385$ & $-24: 04: 01.62$ & 24.221 & 1.280 & 1.990 & 0.065 & 3.1 \\
\hline 4.2 & $04: 16: 08.461$ & $-24: 04: 15.53$ & 9.492 & -12.630 & 1.990 & 0.065 & 3.2 \\
\hline 4.3 & $04: 16: 10.031$ & $-24: 04: 32.62$ & -12.019 & -29.719 & 1.990 & 0.065 & 3.3 \\
\hline 5.1 & 04:16:07.390 & $-24: 04: 02.01$ & 24.157 & 0.890 & 1.990 & 0.065 & 4.1 \\
\hline 5.2 & $04: 16: 08.440$ & $-24: 04: 15.57$ & 9.776 & -12.671 & 1.990 & 0.065 & 4.2 \\
\hline 5.3 & $04: 16: 10.045$ & $-24: 04: 33.03$ & -12.206 & -30.134 & 1.990 & 0.065 & 4.3 \\
\hline 6.1 & $04: 16: 06.618$ & $-24: 04: 21.99$ & 34.731 & -19.086 & 3.223 & 0.065 & 13.1 \\
\hline 6.2 & 04:16:07.709 & $-24: 04: 30.56$ & 19.788 & -27.661 & 3.223 & 0.065 & 13.2 \\
\hline 6.3 & 04:16:09.681 & $-24: 04: 53.53$ & -7.219 & -50.632 & 3.223 & 0.065 & 13.3 \\
\hline 7.1 & $04: 16: 06.297$ & $-24: 04: 27.60$ & 39.130 & -24.700 & 1.637 & 0.065 & 14.1 \\
\hline 7.2 & $04: 16: 07.450$ & $-24: 04: 44.23$ & 23.334 & -41.334 & 1.637 & 0.065 & 14.2 \\
\hline 7.3 & 04:16:08.600 & $-24: 04: 52.76$ & 7.580 & -49.860 & 1.637 & 0.065 & 14.3 \\
\hline 8.1 & $04: 16: 06.246$ & $-24: 04: 37.76$ & 39.818 & -34.861 & 2.302 & 0.065 & 10.1 \\
\hline 8.2 & $04: 16: 06.832$ & $-24: 04: 47.10$ & 31.799 & -44.204 & 2.302 & 0.065 & 10.2 \\
\hline 8.3 & $04: 16: 08.810$ & $-24: 05: 01.93$ & 4.707 & -59.028 & 2.302 & 0.065 & $\mathrm{c} 10.3$ \\
\hline 9.1 & 04:16:05.779 & $-24: 04: 51.22$ & 46.217 & -48.320 & 1.964 & 0.065 & 16.1 \\
\hline 9.2 & 04:16:06.799 & $-24: 05: 04.35$ & 32.249 & -61.452 & 1.964 & 0.065 & 16.2 \\
\hline 9.3 & 04:16:07.586 & $-24: 05: 08.72$ & 21.465 & -65.822 & 1.964 & 0.065 & 16.3 \\
\hline 10.1 & 04:16:05.603 & $-24: 04: 53.70$ & 48.625 & -50.798 & 2.218 & 0.065 & c17.3 \\
\hline 10.2 & $04: 16: 06.866$ & $-24: 05: 09.50$ & 31.331 & -66.598 & 2.218 & 0.065 & c17.2 \\
\hline 10.3 & $04: 16: 07.157$ & $-24: 05: 10.91$ & 27.344 & -68.010 & 2.218 & 0.065 & c17.1 \\
\hline
\end{tabular}

Notes.

a With respect to the luminosity center of G1 and positive in the West and North directions.

b Corresponding image identifier in Zitrin et al. (2013).

with

$$
N_{\text {pos }}=\sum_{j=1}^{N_{\text {sys }}} N_{\text {im }}^{j}=30 .
$$

We adopt a uniform distribution as the prior $P(\boldsymbol{\eta})$ on the parameters.

The source position for each system of multiple images is needed to predict the image positions. For each system, we use the deflection angles of the lens mass model to map the observed image positions to the source plane and take the weighted average of these mapped positions as our source position. Specifically, we weight the mapped source position $\boldsymbol{\beta}_{k}$ by $\sqrt{\mu_{k}} / \sigma_{k}$, where $\mu_{k}$ and $\sigma_{k}$ are the modeled magnification and the positional uncertainty of image $k$, respectively. In other words, we approximate the lensing likelihood as having a delta function at the weighted source position for each image system, thus effectively marginalizing the source position parameters. This approximation works well and is computationally efficient compared to optimizing the source position (e.g., Suyu et al. 2012).

We can either optimize or sample the lens parameters $\eta$ in GLEE. To sample the posterior PDF of $\boldsymbol{\eta}$, we use Markov chain Monte Carlo (MCMC) methods that are based on Dunkley et al.
Table 4

Photometric and Spectroscopic Properties of the Images Magnified but not Multiply Imaged

\begin{tabular}{lccc}
\hline \hline ID & $\begin{array}{c}\text { R.A. } \\
(\text { J2000 })\end{array}$ & $\begin{array}{c}\text { Decl. } \\
(J 2000)\end{array}$ & $z_{\text {sp }}$ \\
\hline s_1 & $04: 16: 08.205$ & $-24: 02: 33.29$ & 2.814 \\
s_2 & $04: 16: 10.781$ & $-24: 03: 27.94$ & 2.807 \\
s_3 & $04: 16: 15.262$ & $-24: 05: 30.90$ & 2.207 \\
\hline
\end{tabular}

(2005) for efficient MCMC sampling and for assessing chain convergence.

\subsection{Mass Components}

\subsubsection{Cluster Members}

The selection of cluster members is a critical step for a reliable gravitational lensing model. In MACS 0416 we have at our disposal already a large set of more than 800 spectroscopically confirmed cluster members, 113 of which are in the HST FoV (63 with WFC3 photometry). This sample is used to identify the locus of the member galaxies, within the HST/WFC3 FoV, in a multi-dimensional color space from 12 CLASH bands. Based on the $n$-dimensional distance of a given galaxy from the 


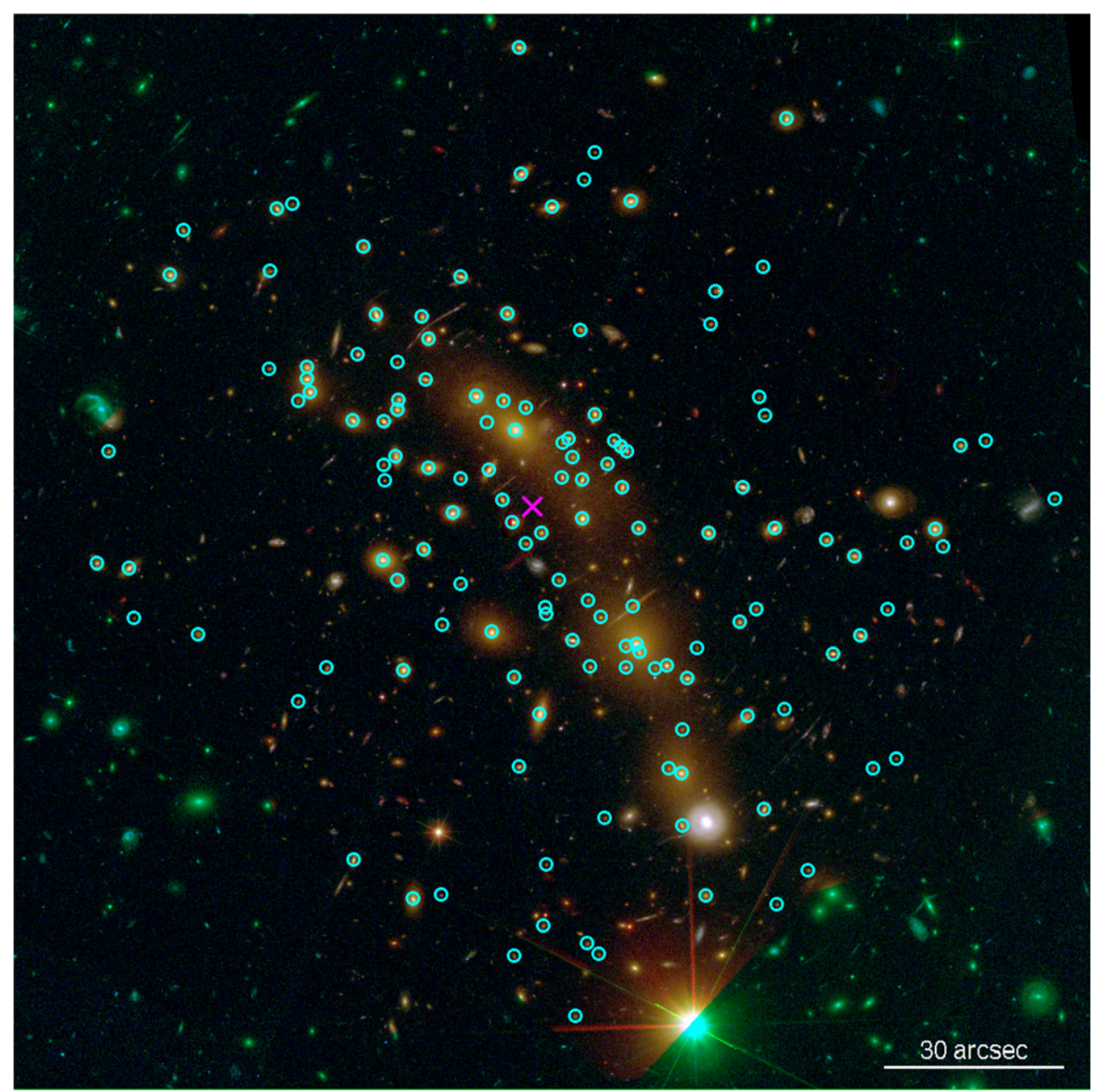

Figure 4. $3^{\prime} \times 3^{\prime}$ color-composite image of MACS 0416 showing in cyan the 175 cluster members, with near-IR F160W magnitudes, selected with the method described in Section 3.3.1, based on spectroscopic and multi-color (12 bands) data, and used in the cluster strong lensing models presented in Section 3.4. The magenta cross locates the cluster luminosity center, estimated by weighting the positions of the candidate cluster members with their F160W magnitudes. North is top and East is left.

color distribution of spectroscopic members, we can assign a membership probability to each galaxy.

Specifically, we first select all galaxies (113) with spectroscopic redshift in the range $0.396 \pm 0.014$, corresponding to $\pm 3000 \mathrm{~km} \mathrm{~s}^{-1}$ rest-frame, and with good photometric data. We exclude the F225W, F275W, F336W, and F390W bands from the CLASH photometric data set due to the low signal-to-noise of these data for the faint member galaxies. We then compute the average colors and the covariance matrix from the color distribution of spectroscopic members using a Minimum Covariance Determinant method (Rousseeuw 1984).

Similarly, we select a representative set of field galaxies with redshifts outside the range associated with cluster members (102) and compute the mean and the covariance matrix of the colors. We assume that the population of cluster members and of field galaxies can each be well described by a multivariate normal distribution with the previously determined averages and covariances. Despite this approximation, we verify a-posteriori that it produces catalogs of cluster members with good purity and completeness.

We tune the member probability threshold in order to maximize the purity of the cluster members, particularly at the brightend of the luminosity function, where the most massive galaxies (those that provide the most important contribution to the mass model of the cluster) reside.
With this method we select 109 members. We find that this represents a pure sample of cluster members, at the expense of some moderate incompleteness. The latter can be significantly alleviated by studying the color-magnitude relation (CMR) of spectroscopic and photometric members. Note that the method outlined above does not use any a priori knowledge of the color-magnitude distribution of galaxies, particularly for specific colors that straddle the $\mathrm{H}+\mathrm{K}$ break and hence produce well distinguished color sequences for cluster galaxies. We therefore supplement the photometric sample obtained from the galaxy distribution in color space with galaxies, fainter than the brightest cluster galaxies, lying on the cluster sequence of the color-magnitude diagram of F606W-F814W versus F606W. We define the mean CMR by using the biweight estimator on spectroscopically confirmed members and select 66 more galaxies lying within a scatter of 0.15 mag from the mean. We verify that the extended sample of cluster galaxies is $\geqslant 95 \%$ complete down to $\mathrm{F} 160 \mathrm{~W}(\mathrm{AB})=21$. We fix our F160W magnitude limit at $24 \mathrm{mag}$, corresponding to approximately $m^{\star}+4.5$ and one magnitude lower than the value of our faintest spectroscopically confirmed cluster member (see Figure 7), beyond which it becomes very difficult to have a reliable estimate of the purity and completeness of the sample. We obtain a final catalog of candidate cluster members containing 175 objects (see Figures 4 and 5). Further details on the selection 


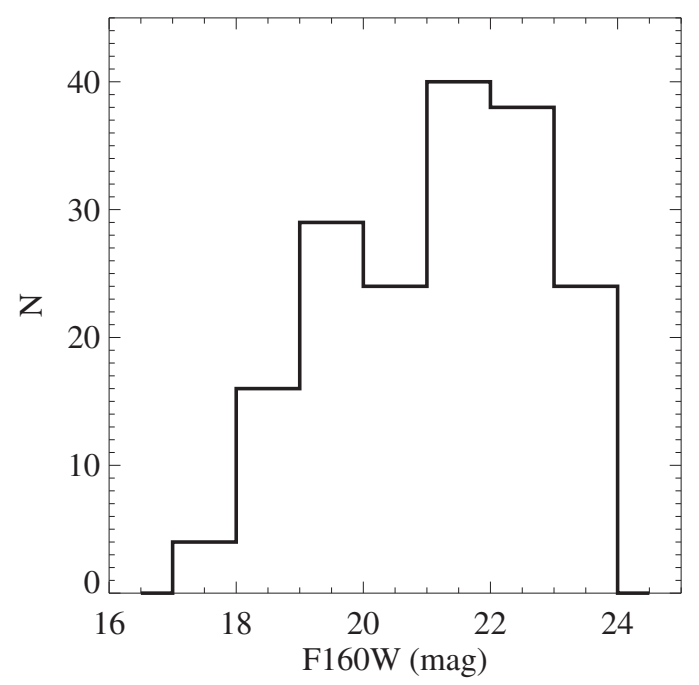

Figure 5. Histogram of the near-IR F160W magnitudes of the 175 candidate cluster members shown in Figure 4.

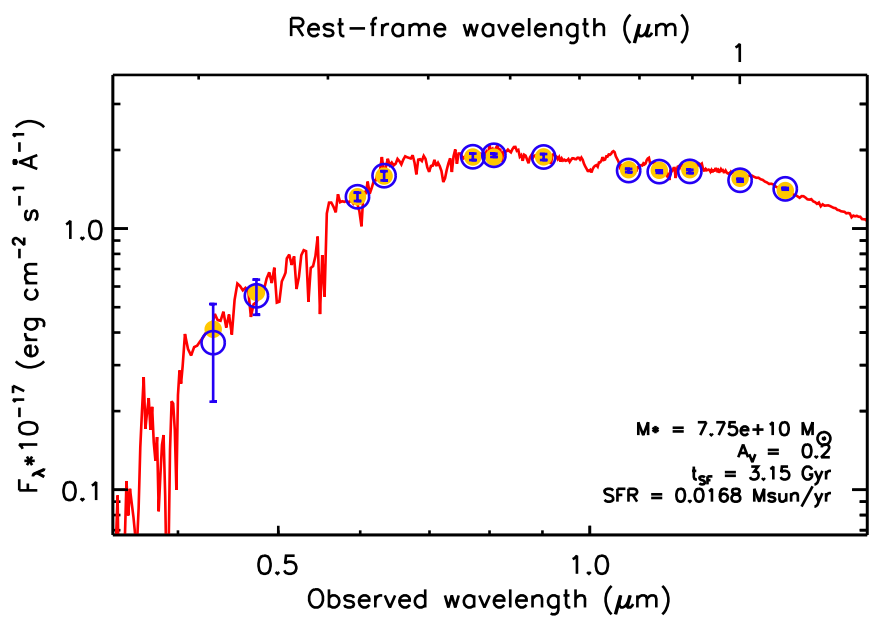

Figure 6. Example of the composite stellar population modeling of the 12 reddest $H S T$ bands of a spectroscopically confirmed galaxy cluster member. We use Bruzual \& Charlot (2003) templates (the best-fitting one is shown here in red) at solar metallicity, with dust and a Salpeter stellar IMF. Observed fluxes with $1 \sigma$ errors are represented with blue empty circles and bars, model-predicted fluxes are shown as orange filled circles.

and statistical analysis of the photometric sample of cluster galaxies will be included in I. Balestra et al. (in preparation).

To determine the mass in the form of stars present in the spectroscopically confirmed cluster members, we fit their spectral energy distributions (SEDs), composed of the 12 reddest HST bands, through composite stellar population models based on Bruzual \& Charlot (2003) templates at solar metallicity and with a Salpeter (1955) stellar initial mass function (IMF). We consider delayed exponential star formation histories and allow for the presence of dust, according to Calzetti et al. (2000) (see also Grillo et al. 2009, 2014). We show a representative example in Figure 6. From Figure 7, we remark that the values of the cluster member stellar masses and F160W magnitudes are very tightly correlated. We find that the best-fitting line is $\log \left(M_{*} / M_{\odot}\right)=18.541-0.416 \times \mathrm{F} 160 \mathrm{~W}$. According to this relation and to the F160W galaxy luminosities, we assign a stellar mass value to each candidate cluster member. Thus, our F160W magnitude limit corresponds to $\log \left(M_{*} / M_{\odot}\right) \simeq 8.6$.

For each cluster galaxy selected, we model its projected dimensionless surface mass density, a.k.a. convergence, as a dual

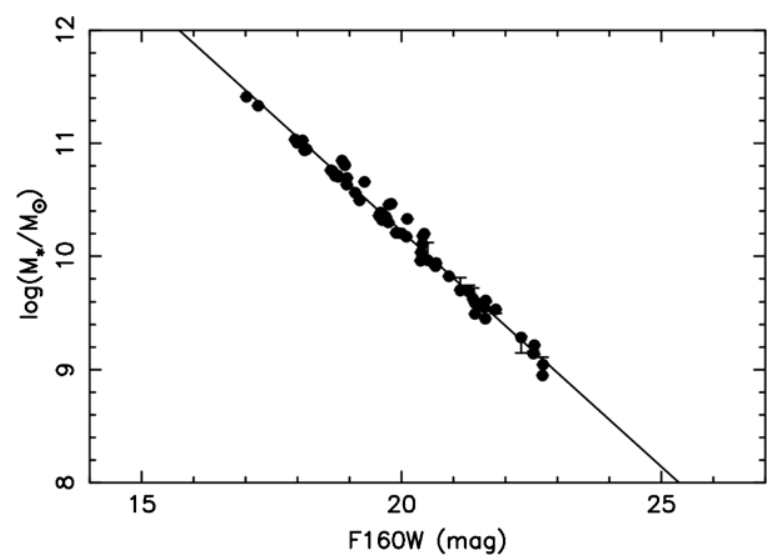

Figure 7. Best-fitting stellar mass values, obtained from the SED modeling of the multicolor HST photometry, as a function of the magnitude values measured in the reddest $H S T / \mathrm{WFC} 3$ broadband $(\mathrm{F} 160 \mathrm{~W})$. The points, with $1 \sigma$ error bars, represent 63 spectroscopically confirmed galaxy cluster members. The solid line shows the best-fitting line.

pseudoisothermal elliptical mass distribution (dPIE; Elíasdóttir et al. 2007; Suyu \& Halkola 2010) with vanishing ellipticity and core radius,

$$
\kappa_{\mathrm{g}}(x, y)=\frac{\vartheta_{\mathrm{E}}}{2}\left(\frac{1}{R}-\frac{1}{\sqrt{R^{2}+r_{\mathrm{t}}^{2}}}\right),
$$

where $(x, y)$ are coordinates on the image/lens plane, $\vartheta_{\mathrm{E}}$ is the cluster galaxy lens strength (a.k.a. Einstein radius), $R\left(=\sqrt{x^{2}+y^{2}}\right)$ is the radial coordinate, and $r_{\mathrm{t}}$ is the "truncation radius." The truncated isothermal distribution is suitably translated by the centroid position of the cluster galaxy luminosity $\left(x_{\mathrm{g}}, y_{\mathrm{g}}\right)$.

The lensing convergence depends in general on the lens and source redshifts. In MACS 0416, there is one lens redshift (that of the galaxy cluster), and various source redshifts of the multiple image systems. The convergence defined in Equation (5) is relative to a background source at redshift $\infty$. For a source at a redshift $z_{\mathrm{s}}$, the convergence associated with that particular source is

$$
\left.\kappa_{\mathrm{g}}\right|_{z=z_{\mathrm{s}}}=\left.\frac{D_{\mathrm{ds}}}{D_{\mathrm{s}}} \kappa_{\mathrm{g}}\right|_{z=\infty},
$$

where $D_{\mathrm{ds}}$ is the angular diameter distance of the source as viewed from the lens, and $D_{\mathrm{s}}$ is the angular diameter distance to the source from us. Therefore, the factor $D_{\mathrm{ds}} / D_{\mathrm{s}}$ is used to relate the deflection angles for the background sources at different redshifts.

For an isothermal profile, we can relate the velocity dispersion of the cluster galaxy to its Einstein radius $\vartheta_{\mathrm{E}}$ via

$$
\sigma / c=\sqrt{\frac{\vartheta_{\mathrm{E}}}{4 \pi}}
$$

where $c$ is the speed of light. Furthermore, the circular velocity of the galaxy, $v_{\mathrm{c}}$, is related to its velocity dispersion $\sigma$ via

$$
v_{\mathrm{c}}=\sqrt{2} \sigma
$$

The three-dimensional mass density distribution corresponding to Equation (5) is

$$
\rho(r) \propto \frac{1}{r^{2}\left(r^{2}+r_{\mathrm{t}}^{2}\right)},
$$


where $r$ is the three-dimensional radius $\left(r=\sqrt{x^{2}+y^{2}+z^{2}}\right)$. Note that for $r \gg r_{\mathrm{t}}$ the mass density distribution scales as $r^{-4}$ and is thus "truncated," and $r_{\mathrm{t}}$ is roughly the half-mass radius (e.g., Elíasdóttir et al. 2007).

\subsubsection{Cluster Dark-matter Halos}

To complete the total mass modeling of the cluster on radial scales larger than those typical of the cluster members, we include two additional mass components. We use two components because the cluster luminosity distribution shows two main peaks. These components are intended to represent the contribution to the total mass budget of all remaining mass (intra cluster light, hot gas and, mainly, dark matter) not associated to the galaxy luminous and dark matter mass distributions. We consider two forms of mass distributions for the cluster mass components: (1) two-dimensional, pseudoisothermal, elliptical (hereafter PIEMD; Kassiola \& Kovner 1993), and (2) three-dimensional, prolate Navarro-Frenk-White (hereafter PNFW; Oguri et al. 2003) mass profiles. Below we describe the convergence relative to a background source at redshift $\infty$; for a source at $z_{\mathrm{s}}$, the convergence is scaled analogously to Equation (6). In Section 3.5, we compare the performances of the two mass models.

The dimensionless surface mass density of PIEMD is of the form

$$
\kappa_{\mathrm{h}}(x, y)=\frac{\vartheta_{\mathrm{E}, \mathrm{h}}}{2 \sqrt{R_{\epsilon}^{2}+r_{\mathrm{c}, \mathrm{h}}^{2}}},
$$

where

$$
R_{\epsilon}^{2}=\frac{x^{2}}{(1+\epsilon)^{2}}+\frac{y^{2}}{(1-\epsilon)^{2}},
$$

$\epsilon$ is the ellipticity defined as $\epsilon \equiv\left(1-q_{\mathrm{h}}\right) /\left(1+q_{\mathrm{h}}\right)$ with $q_{\mathrm{h}}$ being the axis ratio. The strength of the halo is $\vartheta_{\mathrm{E}, \mathrm{h}}$, and the distribution has a central core with radius $r_{\mathrm{c}, \mathrm{h}}$ that marks the transition in the radial dependence in the convergence from $R^{0}$ to $R^{-1}$. The distribution is appropriately translated by the centroid position of the cluster halo $\left(x_{\mathrm{h}}, y_{\mathrm{h}}\right)$ and rotated by the position angle $\phi_{\mathrm{h}}$. Each PIEMD thus requires six parameters to characterize $\left(x_{\mathrm{h}}\right.$, $\left.y_{\mathrm{h}}, q_{\mathrm{h}}, \phi_{\mathrm{h}}, \vartheta_{\mathrm{E}, \mathrm{h}}, r_{\mathrm{c}, \mathrm{h}}\right)$.

The three-dimensional density distribution of PNFW is given by

$$
\rho_{\mathrm{h}}(r)=\frac{\rho_{0, \mathrm{~h}}}{\left(r / r_{\mathrm{s}, \mathrm{h}}\right)\left(1+r / r_{\mathrm{s}, \mathrm{h}}\right)^{2}}
$$

where

$$
r^{2}=c^{2}\left(\frac{x^{2}+y^{2}}{a^{2}}+\frac{z^{2}}{c^{2}}\right), \quad a \leqslant c .
$$

The parameter $a / c$ describes the prolateness of the halo: $a / c=1$ corresponds to a spherical halo, whereas $a / c \ll 0$ corresponds to a highly elongated halo. The orientation of the dark matter halo as seen by a distant observer can be described by two angles: (1) $\varphi_{\mathrm{h}}$, the viewing angle responsible for the level of ellipticity of the two-dimensional projection where $\varphi_{\mathrm{h}}=0$ yield a projected axis ratio of 1 and $\varphi_{\mathrm{h}}=90^{\circ}$ yields the projected axis ratio of $a / c$, and (2) $\phi_{\mathrm{h}}$, the projected major axis position angle. We use the Einstein radius of the cluster halo, $\vartheta_{\mathrm{E}, \mathrm{h}}$, instead of $\rho_{0, \mathrm{~h}}$ to characterize the strength/mass of the halo since strong lensing allows us to measure robustly $\vartheta_{\mathrm{E}, \mathrm{h}}$. We refer to Suyu et al. (2012) for the relation between $\vartheta_{\mathrm{E}, \mathrm{h}}$ and $\rho_{0, \mathrm{~h}}$. In summary, the PNFW is described by seven parameters: $x_{\mathrm{h}}, y_{\mathrm{h}}, a / c, \varphi_{\mathrm{h}}, \phi_{\mathrm{h}}$, $\vartheta_{\mathrm{E}, \mathrm{h}}, r_{\mathrm{s}, \mathrm{h}}$.

\subsection{Mass Models}

In our analysis, we explore different mass models for the galaxy cluster, varying the mass weighting of the cluster members and the mass parameterization of the cluster darkmatter halos.

We start with a model (labeled as 2PIEMD) with only two PIEMD mass profiles (see Section 3.3.2), describing the extended and smooth total mass distribution of the cluster. Then, we add to the two PIEMDs the mass contribution on smaller scales of the 175 cluster members selected in Section 3.3.1. We decide to use their luminosity values, $L$, in the reddest WFC3 band (i.e., the F160W) to assign the relative total mass weights to their dPIE profiles (see Section 3.3.1). In detail, we choose the following scaling relations for the values of the Einstein radius, $\vartheta_{\mathrm{E}, i}$, and truncation radius, $r_{\mathrm{t}, i}$, of the $i$ th cluster member:

$$
\vartheta_{\mathrm{E}, i}=\vartheta_{\mathrm{E}, \mathrm{g}}\left(\frac{L_{i}}{L_{\mathrm{g}}}\right)^{0.5} \text { and } \quad r_{\mathrm{t}, i}=r_{\mathrm{t}, \mathrm{g}}\left(\frac{L_{i}}{L_{\mathrm{g}}}\right)^{0.5} \text {, }
$$

where $\vartheta_{\mathrm{E}, g}$ and $r_{\mathrm{t}, g}$ are two reference values, corresponding, in particular, to those of the brightest cluster galaxy G1. Recalling that for a dPIE profile the total mass, $M_{\mathrm{T}}$, is proportional to the product of the squared value of the effective velocity dispersion, $\sigma$ (where $\sigma \sim \vartheta_{\mathrm{E}}^{0.5}$ ), and the truncation radius, the relations adopted in Equation (14) imply that

$$
\frac{M_{\mathrm{T}, i}}{L_{i}} \sim \frac{\sigma_{i}^{2} r_{\mathrm{t}, i}}{L_{i}} \sim \frac{L_{i}^{0.5} L_{i}^{0.5}}{L_{i}} \sim L_{i}^{0} .
$$

This is therefore equivalent to having cluster members with constant total mass-to-light ratios. We identify this model with 2PIEMD + 175(+1)dPIE $\left(M_{\mathrm{T}} L^{-1}=k\right)$. Note that in this model, as well as in the following ones, we include an extra $(+1) \mathrm{dPIE}$ mass component to take into account the lensing contribution of the bright foreground galaxy (R.A.: 04:16:06.820; decl.: $-24: 05: 08.45 ; z_{\mathrm{sp}}=0.114$, Quality $=3$ ) that is in projection very close to image 10.2 (see Figure 1). We postpone to the future a more complex and rigorous multi-plane lensing analysis and take here into account the different redshift of this particular galaxy through optimizing its effective values of $\vartheta_{\mathrm{E}}$ and $r_{\mathrm{t}}$ without any constraints.

Next, we investigate whether we can find a better lensing model by changing our assumption on the constant total massto-light ratio for the candidate cluster members. In particular, we test the following two relations:

$$
\begin{aligned}
& \vartheta_{\mathrm{E}, i}=\vartheta_{\mathrm{E}, \mathrm{g}}\left(\frac{L_{i}}{L_{\mathrm{g}}}\right)^{0.7} \text { and } \quad r_{\mathrm{t}, i}=r_{\mathrm{t}, \mathrm{g}}\left(\frac{L_{i}}{L_{\mathrm{g}}}\right)^{0.5}, \\
& \vartheta_{\mathrm{E}, i}=\vartheta_{\mathrm{E}, \mathrm{g}}\left(\frac{L_{i}}{L_{\mathrm{g}}}\right)^{0.5} \quad \text { and } \quad r_{\mathrm{t}, i}=r_{\mathrm{t}, \mathrm{g}}\left(\frac{L_{i}}{L_{\mathrm{g}}}\right)^{0.25} .
\end{aligned}
$$

The first one corresponds to values of the total mass-to-light ratio that increase with the luminosity. In particular, we have that

$$
\frac{M_{\mathrm{T}, i}}{L_{i}} \sim \frac{\sigma_{i}^{2} r_{\mathrm{t}, i}}{L_{i}} \sim \frac{L_{i}^{0.7} L_{i}^{0.5}}{L_{i}} \sim L_{i}^{0.2} .
$$

This relation between $M_{\mathrm{T}} / L$ and $L$ is particularly interesting because it has been used to interpret the systematic increase of galaxy effective mass-to-light ratio with effective mass (also known as the tilt of the Fundamental Plane; e.g., Faber et al. 1987; Bender et al. 1992) observed in early-type galaxies. 
Table 5

The Investigated Strong Lensing Models and Their Best-fitting, Minimum- $\chi^{2}$ Values

\begin{tabular}{lr}
\hline \hline Model & $\chi^{2}$ \\
\hline 2PIEMD & 6032 \\
2PIEMD + 175(+1)dPIE $\left(M_{\mathrm{T}} L^{-1}=k\right)$ & 1169 \\
2PIEMD + 175(+1)dPIE $\left(M_{\mathrm{T}} L^{-1} \sim L^{0.2}\right)$ & 915 \\
2PIEMD + 175(+1)dPIE $\left(r_{\mathrm{t}} \sim \sigma\right)$ & 1262 \\
2PNFW & 6973 \\
2PNFW + 175(+1)dPIE $\left(M_{\mathrm{T}} L^{-1}=k\right)$ & 1767 \\
2PNFW + 175(+1)dPIE $\left(M_{\mathrm{T}} L^{-1} \sim L^{0.2}\right)$ & 1529 \\
2PNFW + 175(+1)dPIE $\left(r_{\mathrm{t}} \sim \sigma\right)$ & 1901 \\
\hline
\end{tabular}

The second one instead is motivated by theoretical studies (e.g., Merritt 1983) that predict a linear relation between truncation radius and velocity dispersion for galaxies residing in a cluster environment. We refer to these two models as 2PIEMD + $175(+1) \mathrm{dPIE}\left(M_{\mathrm{T}} L^{-1} \sim L^{0.2}\right)$ and 2PIEMD $+175(+1) \mathrm{dPIE}$ $\left(r_{\mathrm{t}} \sim \sigma\right)$, respectively.

Finally, we try four additional models, analogous to the previous ones, in which only the mass distribution of the two extended cluster dark-matter halos is substituted with PNFW profiles (see Section 3.3.2). We label these models as 2PNFW, $2 \mathrm{PNFW}+175(+1) \mathrm{dPIE}\left(M_{\mathrm{T}} L^{-1}=k\right), 2 \mathrm{PNFW}+$ $175(+1) \mathrm{dPIE}\left(M_{\mathrm{T}} L^{-1} \sim L^{0.2}\right)$, and $2 \mathrm{PNFW}+175(+1) \mathrm{dPIE}$ $\left(r_{\mathrm{t}} \sim \sigma\right)$, respectively.

\subsection{Results}

We show the best-fitting, minimum- $\chi^{2}$ values of the eight different mass models in Table 5. First, we notice that the inclusion of the cluster members results in $\chi^{2}$ values that are always more than a factor of three lower than those obtained with only the two extended cluster dark-matter halos. Then, we find that the models with scaling of the galaxy total mass-to-light ratio increasing with the luminosity are slightly better in reproducing the observed multiple image systems. Interestingly, we also see that there is significant evidence that cored elliptical pseudo-isothermal profiles are better-suited than three-dimensional, PNFW profiles to represent the extended total mass distribution of MACS 0416; considering the models with the cluster member contribution, the $\chi^{2}$ values of the prolate NFW halos are more than $50 \%$ higher than those of the PIEMD halos, despite the prolate NFW halos having more parameters.

In summary, we conclude that the mass model of MACS 0416 that best fits the strong lensing observables is composed of 2 cored elliptical pseudo-isothermal mass distributions and numerous (175) dual pseudo-isothermal mass distributions, scaled with total mass-to-light ratios increasing with the nearIR luminosities of the candidate cluster members. We confirm that detailed modeling on the small mass/radial scales of the many cluster galaxies is fundamental to a precise multiple image reconstruction.

\subsubsection{The Best-fitting Model}

The best-fitting model, with a minimum $\chi^{2}$ value of 915 (see Table 5), can reproduce the multiple images of the 10 strong lensing systems very accurately, with a median (rms) offset between the observed and model-predicted positions of only 0 '.31 (0.'36), i.e., approximately 5 (6) pixels. In Figure 8 , we compare the positions of the multiple images measured and listed in Table 3 (indicated by circles) with those reconstructed
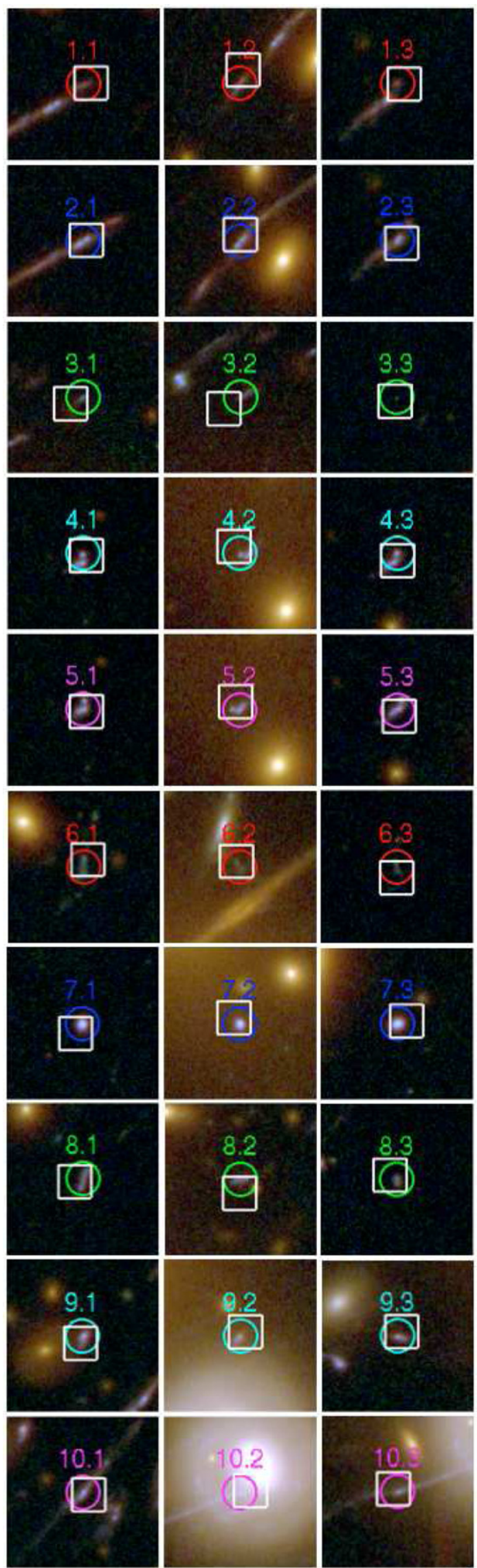

Figure 8. Snapshots (6 arcsec across) of the 10 strong lensing systems with spectroscopic redshifts. The observed and predicted (by the best-fitting model; see Table 5) positions of the multiple images are marked, respectively, with colored circles and white squares. 


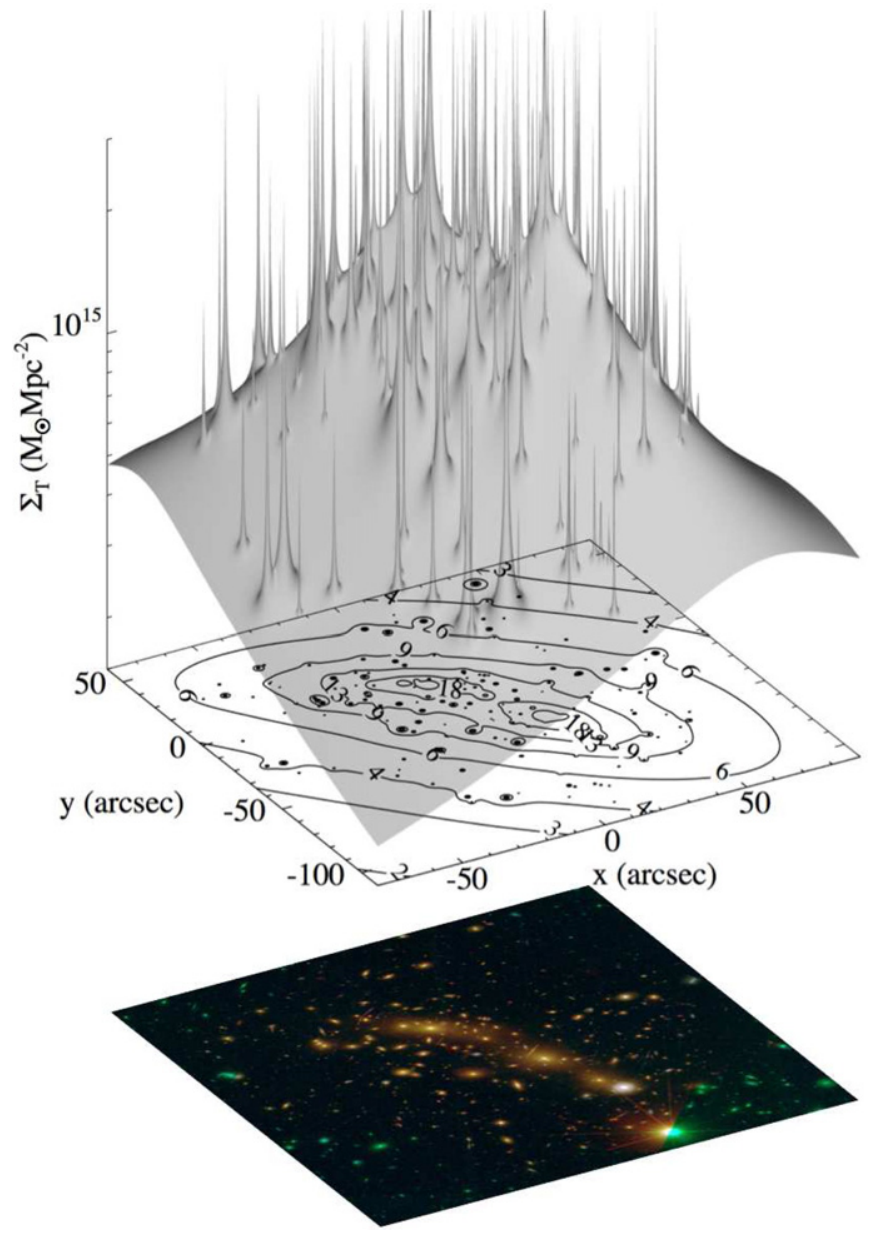

Figure 9. Total surface mass density $\Sigma_{\mathrm{T}}$ in the inner regions of MACS 0416 reconstructed from the best-fitting strong lensing model (see Table 5). The different contributions of the two extended dark-matter halo and many candidate cluster member components are visible. The contour levels on the lens plane are in units of $10^{14} M_{\odot} \mathrm{Mpc}^{-2}$.

according to our model (squares). We notice that every system is almost perfectly reconstructed, without any systematic offset in the predicted image positions. This implies that the expected complex total mass distribution of the cluster is globally described very well by our simple parameterized mass profiles.

In Figures 9 and 10, we show the reconstructed surface mass density of MACS 0416. We illustrate the total mass density, $\Sigma_{\mathrm{T}}$, the smooth and extended contribution of the cluster darkmatter halos, $\Sigma_{\mathrm{H}}$, and the more concentrated and localized mass density of the cluster members, $\Sigma_{\mathrm{G}}$. We remark that the cluster dark-matter halo components are traced reasonably well by the total light distribution of the cluster.

Finally, we calculate the magnification factor values, $\mu$, in the central regions of the cluster for several redshifts $z_{\mathrm{s}}$ of a possible background source and show them in Figure 11. In Table 6 and Figure 12, we also show the sizes of the area $A$ inside which the magnification factor is within the tabulated ranges. We remark that MACS 0416 is an efficient deflector with extended regions of high magnification. More quantitatively, by looking at the values of $A(\mu<0)$, corresponding approximately to the surface enclosed within the tangential critical curve, we notice that the size of this area is enlarged by a factor of 1.5 when the source redshift is increased from 2 to 10 . Varying the source redshift within the same range, the area with the largest magnification factors, $A(|\mu| \geqslant 30)$, grows by a factor of 1.3 . Moreover, raising
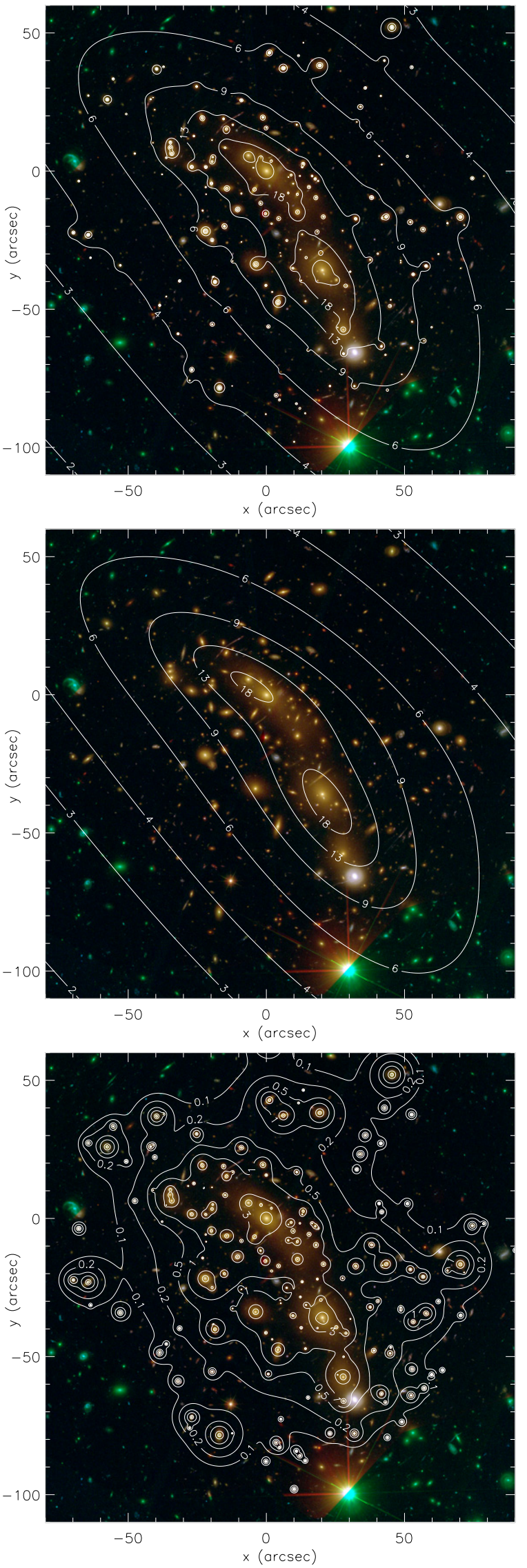

Figure 10. Decomposition of the reconstructed total surface mass density, $\Sigma_{\mathrm{T}}$, (on the top) into the surface mass densities of the two extended cluster darkmatter halos, $\Sigma_{\mathrm{H}}$, (in the middle) and many candidate cluster members, $\Sigma_{\mathrm{G}}$ (on the bottom). The contour levels on the lens plane are in units of $10^{14} \mathrm{M}_{\odot} \mathrm{Mpc}^{-2}$. 

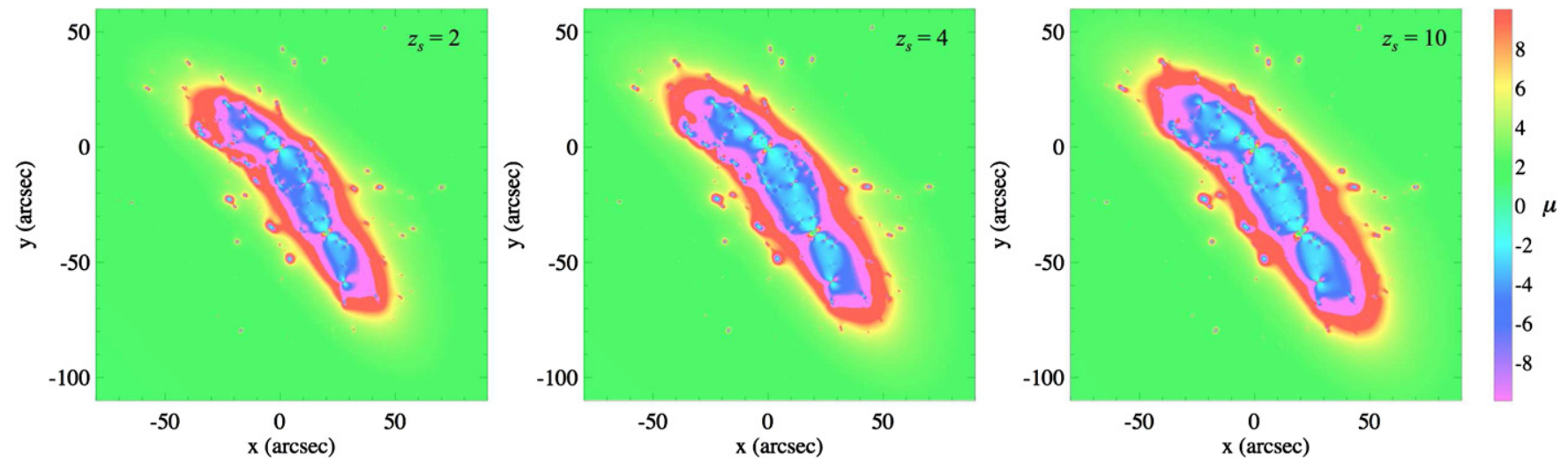

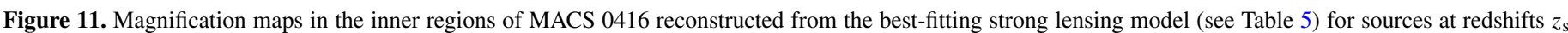

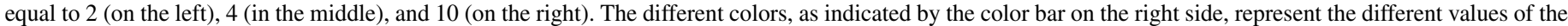
magnification factor $\mu$ on a linear scale extending from -10 to 10 .

Table 6

Values of the Area $A$ on the Lens Plane Where the Magnification Factor $\mu$ is Included in the Specified Ranges for Different Source Redshifts $z_{\mathrm{s}}$

\begin{tabular}{lccccc}
\hline \hline & $\begin{array}{c}A(\mu<0) \\
\left(\operatorname{arcmin}^{2}\right)\end{array}$ & $\begin{array}{c}A(3 \leqslant|\mu|<5) \\
\left(\operatorname{arcmin}^{2}\right)\end{array}$ & $\begin{array}{c}A(5 \leqslant|\mu|<10) \\
\left(\operatorname{arcmin}^{2}\right)\end{array}$ & $\begin{array}{c}A(10 \leqslant|\mu|<30) \\
\left(\operatorname{arcmin}^{2}\right)\end{array}$ & $\begin{array}{c}A(|\mu| \geqslant 30) \\
\left(\operatorname{arcmin}^{2}\right)\end{array}$ \\
\hline$z_{\mathrm{s}}=2$ & 0.57 & 0.88 & 0.55 & 0.34 & 0.17 \\
$z_{\mathrm{s}}=3$ & 0.70 & 1.01 & 0.62 & 0.40 & 0.20 \\
$z_{\mathrm{s}}=4$ & 0.77 & 1.07 & 0.67 & 0.42 & 0.22 \\
$z_{\mathrm{s}}=6$ & 0.84 & 1.15 & 0.71 & 0.46 & 0.23 \\
$z_{\mathrm{s}}=8$ & 0.88 & 1.19 & 0.73 & 0.48 & 0.23 \\
$z_{\mathrm{s}}=10$ & 0.90 & 1.22 & 0.74 & 0.50 & 0.23 \\
\hline
\end{tabular}

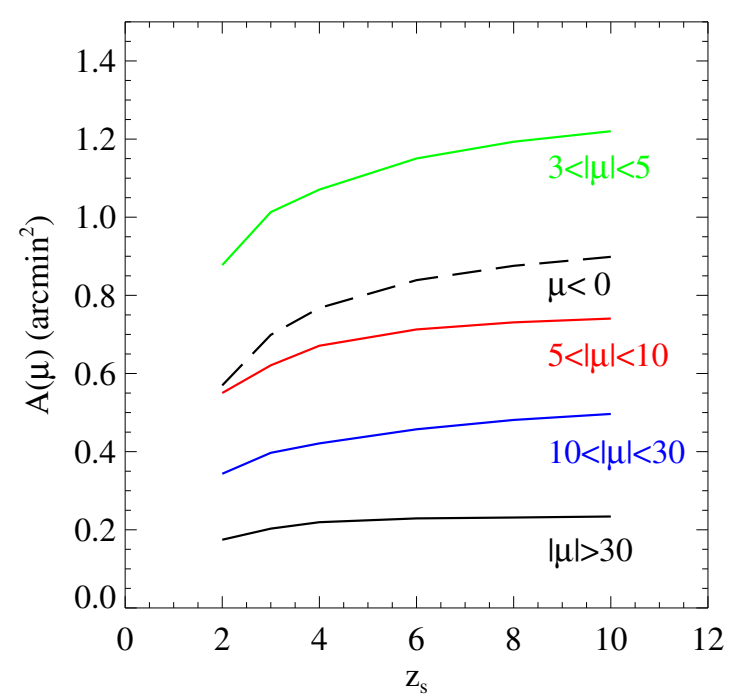

Figure 12. Values of the area $A$ on the lens plane where the magnification factor $\mu$ is included in the specified ranges for different source redshifts $z_{\mathrm{s}}$ (see also Table 6).

the value of $z_{\mathrm{s}}$ from 2 to 10 also increases the percentage of surface of the lens plane with medium magnification values.

\subsubsection{MCMC Analysis}

As mentioned in Section 3.2, we apply a MCMC technique to sample the posterior PDF of the parameters $\eta$ of the best-fitting 2 PIEMD $+175(+1) \mathrm{dPIE}\left(M_{\mathrm{T}} L^{-1} \sim L^{0.2}\right)$ model. The uncertainties obtained from a MCMC analysis for the parameters of a model, and thus for the quantities derived from them, correlate with the uncertainties assigned to the observables. To get realistic uncertainties for the model parameters, the uncertainties of the observables have to be scaled so that the value of the bestfitting $\chi^{2}$ is comparable to the number of degrees of freedom of the investigated system (in other words, the reduced $\chi^{2}$ value should be approximately equal to 1 ). For this reason, in our bestfitting strong lensing model we increase the positional error of the observed multiple images $\delta_{x, y}$ by a factor of approximately six, i.e., to 0.'4. This can account for, e.g., line-of-sight structures and small dark-matter clumps that are not encapsulated in our mass model. In this way, the value (24) of the $\chi^{2}$ is comparable to the number (24) of the degrees of freedom. The latter is given by the number of lensing observables ( $x$ and $y$ of each multiple image) minus the number of parameters $\eta$ of the model ( $x$ and $y$ of each source, $x_{\mathrm{h}}, y_{\mathrm{h}}, q_{\mathrm{h}}, \phi_{\mathrm{h}}, \vartheta_{\mathrm{E}, \mathrm{h}}$, and $r_{\mathrm{c}, \mathrm{h}}$ of each cluster dark-matter halo, $\vartheta_{\mathrm{E}, \mathrm{g}}$ and $r_{\mathrm{t}, \mathrm{g}}$ of the scaling relations of the cluster members, and $\vartheta_{\mathrm{E}}$ and $r_{\mathrm{t}}$ of the foreground galaxy). We show the results, derived from a chain with $2 \times 10^{6}$ samples (with an acceptance rate of approximately 0.22), in Figure 13 and Table 7.

Looking at Figure 13, we observe that the values of $x_{\mathrm{h}}$ and $y_{\mathrm{h}}$ of each of the two cluster dark-matter halos are anticorrelated. This means that, to preserve the goodness of the fit, shifts of the mass centers of these two components are only allowed in the northeast (or southwest) direction. As expected, for the same mass components, we also find that the values of $\vartheta_{\mathrm{E}, \mathrm{h}}$, and $r_{\mathrm{c}, \mathrm{h}}$ are correlated. From Equation (10), it is clear that in order to obtain a fixed amount of projected mass within a given small $\left(R \lesssim r_{\mathrm{c}, \mathrm{h}}\right)$ circle, an increase in the value of $\vartheta_{\mathrm{E}, \mathrm{h}}$ must be counterbalanced by a suitable increase in the value of $r_{\mathrm{c}, \mathrm{h}}$. Moreover, the values of the strength of the dark-matter halos, $\vartheta_{\mathrm{E}, \mathrm{h} 1}$ and $\vartheta_{\mathrm{E}, \mathrm{h} 2}$, are anticorrelated. This follows from the fact that the contributions of the two halos to the total mass in the central regions of the cluster (tightly constrained by the multiple image systems) compensate each other. Not surprisingly, we do 


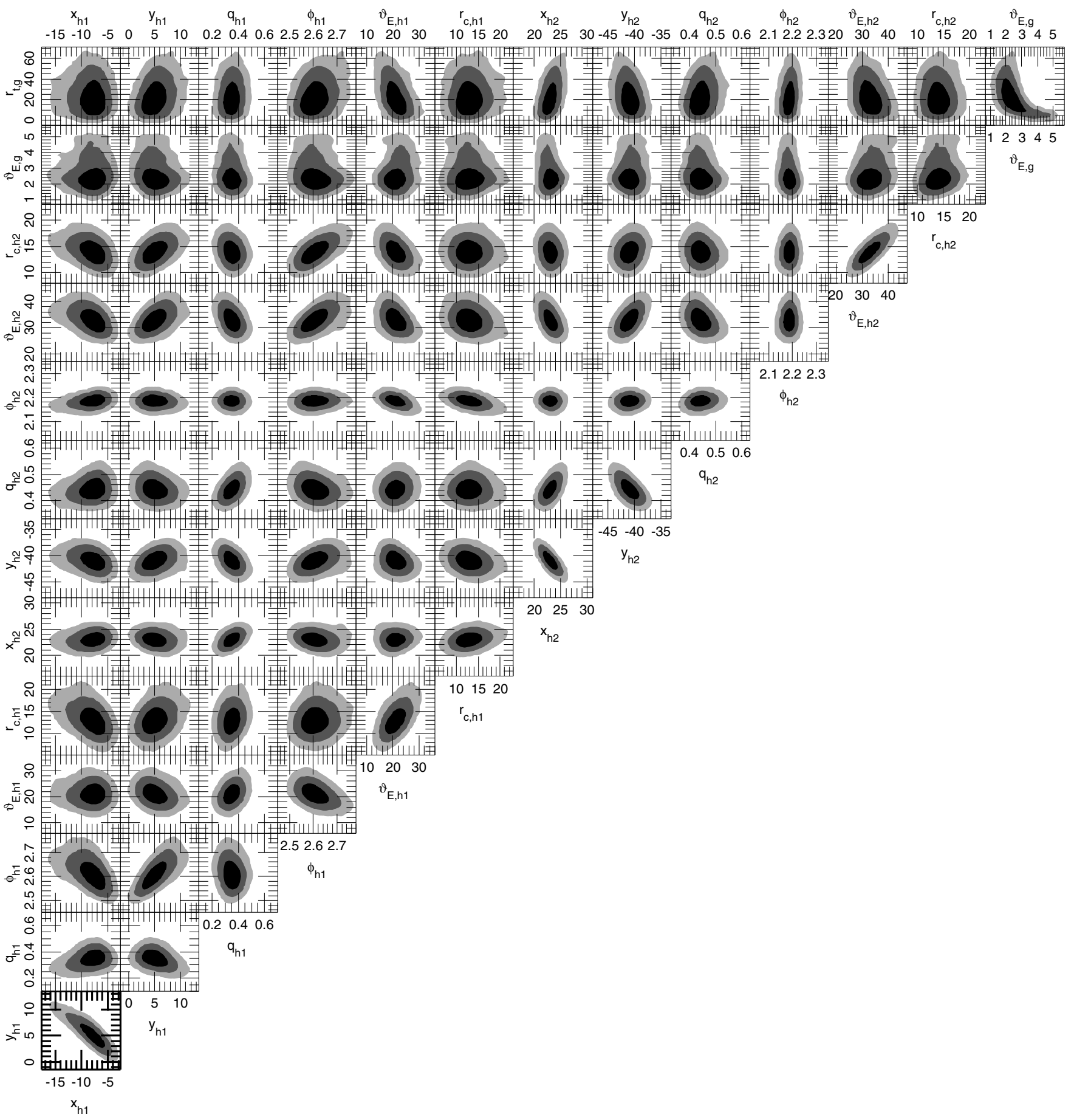

Figure 13. Estimates of the uncertainties and correlations of the parameters $\eta$ of the 2PIEMD $+175(+1) \mathrm{dPIE}\left(M_{\mathrm{T}} L^{-1} \sim L^{0.2}\right)$ model. The gray contour levels on the planes represent the $1 \sigma, 2 \sigma$, and $3 \sigma$ confidence regions and are obtained from a MCMC chain with $2 \times 10^{6}$ samples.

not have much information about the values of the truncation radius $r_{\mathrm{t}, \mathrm{g}}$ of the cluster members. In Figure 1, we see indeed that none of the lensing systems has two or more multiple images located close to and around a single cluster member. This would have enabled a determination of the values of both $\vartheta_{\mathrm{E}, \mathrm{g}}$ and $r_{\mathrm{t}, \mathrm{g}}$. In our model, the total mass profiles of the cluster members are thus well approximated by simple isothermal mass distributions (see Equation (5) for large values of $r_{\mathrm{t}, \mathrm{g}}$ ).

From Tables 1 and 7 and Figure 14, we remark that the reconstructed centers of two cluster dark-matter halos are significantly separated, more than $3 \sigma$ away, from the centers of the two brightest cluster galaxies. In detail, the northern halo is at a projected distance of approximately $50 \mathrm{kpc}$ from G1 and a smaller projected distance of approximately $30 \mathrm{kpc}$ separates the centers of the southern halo and G2. Interestingly, the northern halo is preferentially displaced toward the northeast direction, whereas the southern halo toward the southwest direction, resulting in a projected distance between the two cluster darkmatter halos of approximately $300 \mathrm{kpc}$. We have checked that (1) fixing the halo centers to those of the brightest cluster galaxies results in $\chi^{2}$ values that are approximately a factor of four higher than those obtained with the halo centers free to vary, and 
Table 7

Median Values and Intervals at $1 \sigma, 2 \sigma$, and $3 \sigma$ Confidence Level of the Parameters $\eta$ of the 2PIEMD $+175(+1) \operatorname{dPIE}\left(M_{\mathrm{T}} L^{-1} \sim L^{0.2}\right)$ Model

\begin{tabular}{|c|c|c|c|c|}
\hline & Median & $1 \sigma \mathrm{CL}$ & $2 \sigma \mathrm{CL}$ & $3 \sigma \mathrm{CL}$ \\
\hline$x_{\mathrm{h} 1}\left({ }^{\prime \prime}\right)$ & -7.8 & $\begin{array}{l}+1.5 \\
-1.8\end{array}$ & $\begin{array}{l}+2.8 \\
-4.4\end{array}$ & $\begin{array}{l}+3.9 \\
-7.5\end{array}$ \\
\hline$y_{\mathrm{h} 1}\left({ }^{\prime \prime}\right)$ & 5.0 & $\begin{array}{l}+1.7 \\
-1.5\end{array}$ & $\begin{array}{l}+3.6 \\
-2.9\end{array}$ & $\begin{array}{l}+5.7 \\
-4.2\end{array}$ \\
\hline$q_{\mathrm{h} 1}$ & 0.35 & $\begin{array}{l}+0.04 \\
-0.04\end{array}$ & $\begin{array}{l}+0.08 \\
-0.08\end{array}$ & $\begin{array}{l}+0.13 \\
-0.12\end{array}$ \\
\hline$\phi_{\mathrm{h} 1}(\mathrm{rad})$ & 2.61 & $\begin{array}{l}+0.04 \\
-0.04\end{array}$ & $\begin{array}{l}+0.09 \\
-0.07\end{array}$ & $\begin{array}{l}+0.14 \\
-0.10\end{array}$ \\
\hline$\vartheta_{\mathrm{E}, \mathrm{h} 1}\left({ }^{\prime \prime}\right)$ & 21.0 & $\begin{array}{l}+2.7 \\
-2.5\end{array}$ & $\begin{array}{l}+5.4 \\
-4.8\end{array}$ & $\begin{array}{l}+8.6 \\
-7.1\end{array}$ \\
\hline$r_{\mathrm{c}, \mathrm{h} 1}\left({ }^{\prime \prime}\right)$ & 12.9 & $\begin{array}{l}+2.1 \\
-1.9\end{array}$ & $\begin{array}{l}+4.5 \\
-3.8\end{array}$ & $\begin{array}{l}+7.5 \\
-5.7\end{array}$ \\
\hline$x_{\mathrm{h} 2}\left({ }^{\prime \prime}\right)$ & 23.0 & $\begin{array}{l}+0.9 \\
-0.9\end{array}$ & $\begin{array}{l}+1.8 \\
-1.7\end{array}$ & $\begin{array}{l}+2.7 \\
-2.4\end{array}$ \\
\hline$y_{\mathrm{h} 2}\left({ }^{\prime \prime}\right)$ & -40.9 & $\begin{array}{l}+1.2 \\
-1.2\end{array}$ & $\begin{array}{l}+2.4 \\
-2.3\end{array}$ & $\begin{array}{l}+3.5 \\
-3.4\end{array}$ \\
\hline$q_{\mathrm{h} 2}$ & 0.44 & $\begin{array}{l}+0.03 \\
-0.02\end{array}$ & $\begin{array}{l}+0.06 \\
-0.05\end{array}$ & $\begin{array}{l}+0.09 \\
-0.07\end{array}$ \\
\hline$\phi_{\mathrm{h} 2}(\mathrm{rad})$ & 2.19 & $\begin{array}{l}+0.01 \\
-0.01\end{array}$ & $\begin{array}{l}+0.03 \\
-0.03\end{array}$ & $\begin{array}{l}+0.04 \\
-0.05\end{array}$ \\
\hline$\vartheta_{\mathrm{E}, \mathrm{h} 2}\left({ }^{\prime \prime}\right)$ & 32.8 & $\begin{array}{l}+3.1 \\
-2.8\end{array}$ & $\begin{array}{l}+6.3 \\
-5.2\end{array}$ & $\begin{array}{l}+9.5 \\
-7.4\end{array}$ \\
\hline$r_{\mathrm{c}, \mathrm{h} 2}\left({ }^{\prime \prime}\right)$ & 14.0 & $\begin{array}{l}+1.5 \\
-1.4\end{array}$ & $\begin{array}{l}+3.0 \\
-2.7\end{array}$ & $\begin{array}{l}+4.8 \\
-4.0\end{array}$ \\
\hline$\vartheta_{\mathrm{E}, \mathrm{g}}\left({ }^{\prime \prime}\right)$ & 2.3 & $\begin{array}{l}+0.6 \\
-0.4\end{array}$ & $\begin{array}{l}+1.8 \\
-0.7\end{array}$ & $\begin{array}{l}+2.6 \\
-1.1\end{array}$ \\
\hline$r_{\mathrm{t}, \mathrm{g}}\left({ }^{\prime \prime}\right)$ & 21 & $\begin{array}{l}+13 \\
-10\end{array}$ & $\begin{array}{l}+27 \\
-16\end{array}$ & $\begin{array}{l}+39 \\
-17\end{array}$ \\
\hline
\end{tabular}

Notes. The parameters $q_{\mathrm{h} 1}$ and $q_{\mathrm{h} 2}$ are the axis ratios of the two cluster darkmatter halos (introduced in Section 3.3.2). The angles $\phi_{\mathrm{h} 1}$ and $\phi_{\mathrm{h} 2}$ are measured counterclockwise from the positive $x$ axis (West).

(2) increasing the F160W magnitude value of G1 by 0.3 mag, i.e., mimicking an overestimate of the galaxy luminosity due to a possible contamination from the intra cluster light, reduces the offset between the centers of the northern halo and G1 by only about $10 \%$. As a result of the superposition of two components, we mention that the density peaks of the two superposed dark-matter clumps are less distant than the individual dark-matter halo centers from the centers of the brightest cluster galaxies (see Figure 10). Furthermore, we notice that within the adopted mass parameterization, the two dark-matter components require appreciably large core radii, at more than $3 \sigma \mathrm{CL}$. The median values of the two cores are of 69 and $75 \mathrm{kpc}$ for the northern and southern halos, respectively.

Next, we extract from the MCMC chain 100 different models to quantify the statistical uncertainty on the derived circular quantities of average surface mass density

$$
\Sigma(<R) \equiv \frac{\int_{0}^{R} \Sigma(\tilde{R}) 2 \pi \tilde{R} \mathrm{~d} \tilde{R}}{\pi \tilde{R}^{2}}
$$

and cumulative projected mass

$$
M(<R) \equiv \int_{0}^{R} \Sigma(\tilde{R}) 2 \pi \tilde{R} \mathrm{~d} \tilde{R}
$$

where $\tilde{\boldsymbol{R}}=\tilde{R} \boldsymbol{e}_{\tilde{\boldsymbol{R}}}=(x, y)$ and $\boldsymbol{e}_{\tilde{\boldsymbol{R}}}=\tilde{\boldsymbol{R}} / \tilde{R}$. As done in the previous section, we decompose the total $\Sigma(<R)$ and $M(<R)$ into their cluster dark-matter halo and cluster member components. We calculate the distances $R$ on the lens plane from the barycenter, or center of mass, of the cluster

$$
\boldsymbol{R}_{\mathrm{b}} \equiv \frac{\int \Sigma_{\mathrm{T}}(\tilde{\boldsymbol{R}}) \tilde{\boldsymbol{R}} \mathrm{d} \tilde{\boldsymbol{R}}}{\int \Sigma_{\mathrm{T}}(\tilde{\boldsymbol{R}}) \mathrm{d} \tilde{\boldsymbol{R}}} .
$$

According to our best-fitting model, we find that the coordinates of the barycenter, with respect to the luminosity center of $\mathrm{G} 1$, in arcsec are $(8.14,-22.22)$. Therefore, considering the line that connects the luminosity centers of $G 1$ and $G 2$, the

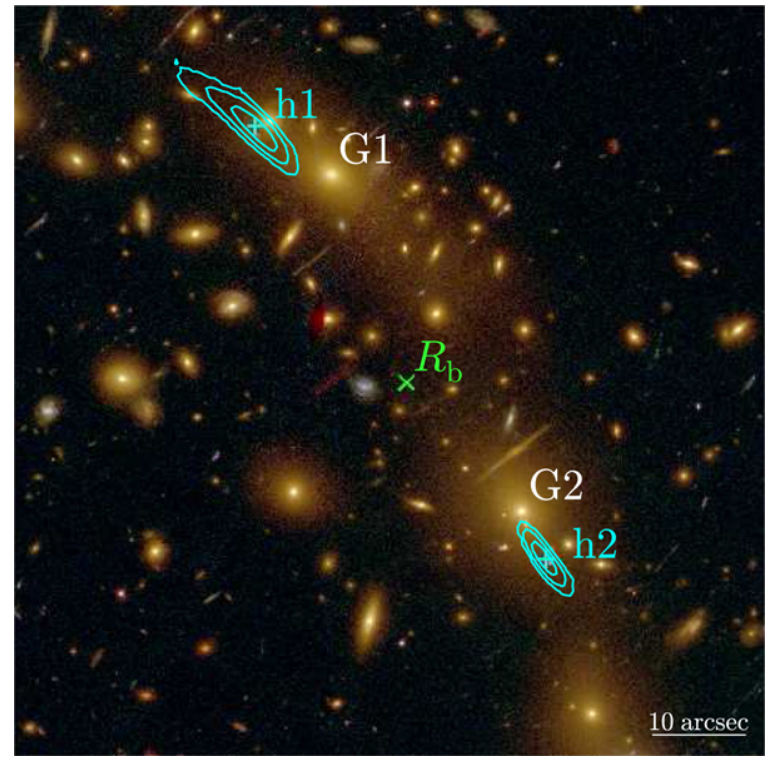

Figure 14. $80^{\prime \prime} \times 80^{\prime \prime}$ cluster-core image showing the positions of the centers of the two extended dark-matter halos (h1 and h2, cyan plus symbols, see also Table 7) and of the cluster barycenter $\left(\boldsymbol{R}_{\mathrm{b}}\right.$, green cross, defined in Equation (21)) of the best-fitting lens model. Given the uncertainties shown in Figure 13, reproduced here with cyan contours, the dark-matter-halo centers are offset from the luminosity centers of the closest brightest cluster galaxies (G1 and G2) at more than $3 \sigma \mathrm{CL}$. North is up and east is left.

center of mass of MACS 0416 lies on the eastern side (see Figure 14), where more luminous cluster members are observed (see Figure 1). Figure 15 illustrates the radial dependence of the functions $\Sigma(<R)$ and $M(<R)$.

We notice that the cluster member and dark-matter halo components have remarkably similar distributions. Both $\Sigma_{\mathrm{G}}(<R)$ and $\Sigma_{\mathrm{H}}(<R)$ show very flat inner profiles, with core radii of approximately $100 \mathrm{kpc}$. The small bump in the cluster member component, visible at about $130 \mathrm{kpc}$, is due to the presence of the two brightest cluster galaxies G1 and G2 at such a projected distance from the barycenter. It is also interesting to remark that the cluster-galaxy and cluster-halo mass components must be anticorrelated. In fact, they clearly have relative uncertainties that are larger than those of the total quantities. At $1 \sigma \mathrm{CL}$, the cumulative projected total mass profile exhibits, surprisingly, nearly constant uncertainty of a few per cent over the investigated radial range extending from 10 to $350 \mathrm{kpc}$. At more than $100 \mathrm{kpc}$ in projection from the barycenter, we measure a cluster member over total mass ratio, $M_{\mathrm{G}} / M_{\mathrm{T}}(<R)$, of $13_{-4}^{+5} \%$.

In Figure 16, we plot the cumulative projected total mass profile resulting from our best-fitting strong lensing model and that from the independent weak lensing analysis by Umetsu et al. (2014). It is well known that the strong and weak lensing effects allow one to map the projected total mass of a cluster on different radial scales. We show here that the estimates of the two total mass diagnostics overlap between approximately 300 and $400 \mathrm{kpc}$ from the center of MACS 0416 and over this radial range they are consistent, given the $1 \sigma$ uncertainties, despite the slightly different definition of the cluster center in the two studies. We remark that such a good agreement between the strong and weak lensing mass estimates has been observed only in a few galaxy clusters (e.g., Umetsu et al. 2011, 2012; Coe et al. 2012; Eichner et al. 2013; Medezinski et al. 2013), partly due to the different systematic uncertainties affecting the two methods and to the not always optimal quality of the data available. 

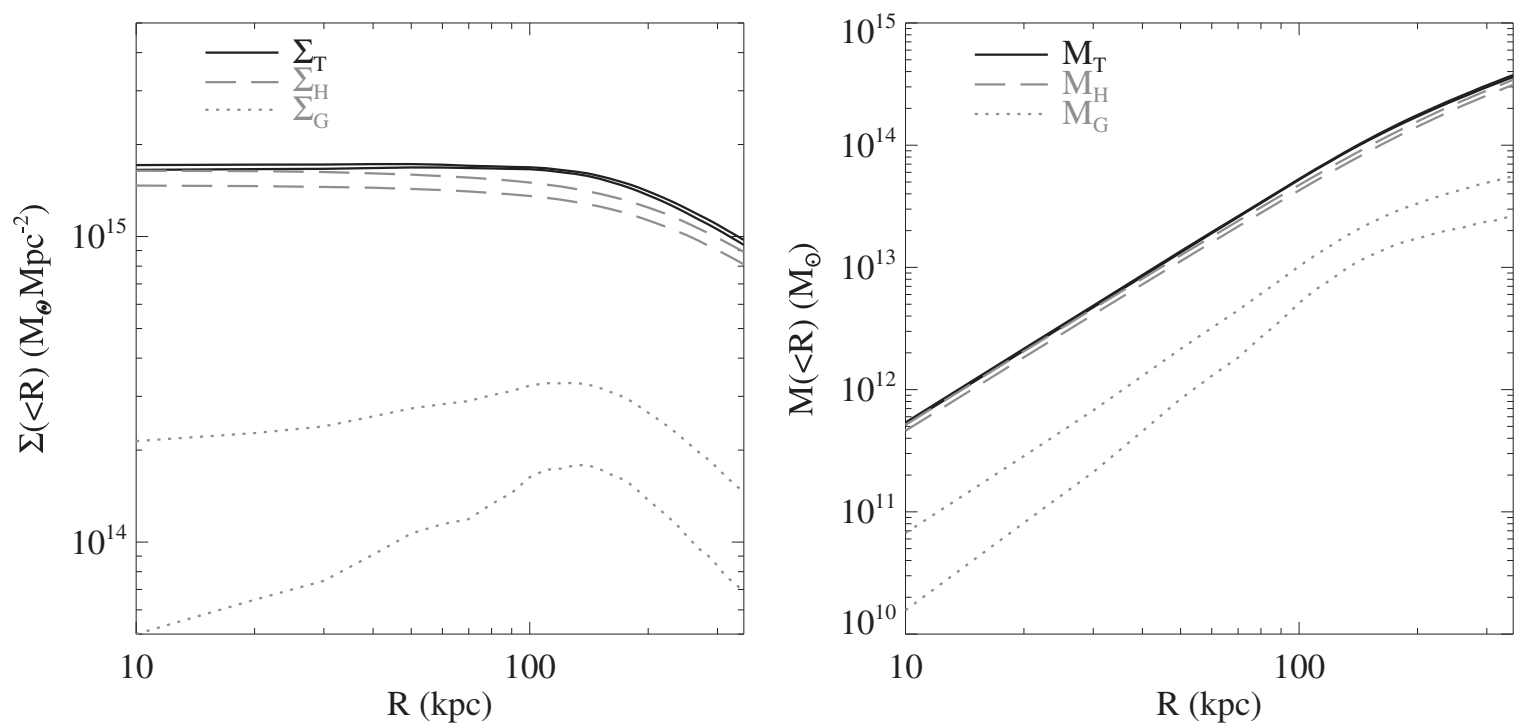

Figure 15. Average surface mass density, $\Sigma(<R)$, and cumulative projected mass, $M(<R)$, profiles of the 2PIEMD $+175(+1) \mathrm{dPIE}\left(M_{\mathrm{T}} L^{-1} \sim L^{0.2}\right)$ model. The solid, long-dashed, and dotted lines represent, respectively, the total, cluster dark-matter halo, and cluster member profiles at $1 \sigma$ confidence level.

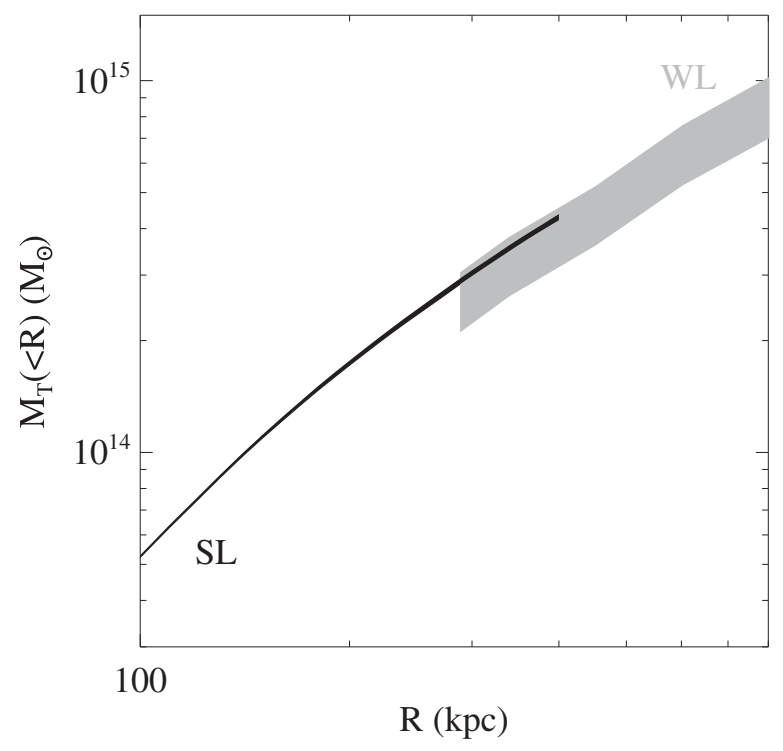

Figure 16. Cumulative projected total mass, $M_{\mathrm{T}}(<R)$, profiles obtained from the strong lensing (SL) analysis presented in this work, i.e., from the 2PIEMD + $175(+1) \mathrm{dPIE}\left(M_{\mathrm{T}} L^{-1} \sim L^{0.2}\right)$ model, and from the weak lensing (WL) analysis presented in Umetsu et al. (2014). Intervals are at $1 \sigma$ confidence level.

We give an estimate of the systematic errors in our surface mass density and cumulative projected mass profiles by considering the best-fitting results of the six strong lensing models, shown in Table 5, that include the mass contribution of the galaxy cluster members. We compare the model-reconstructed quantities in Figure 17. Surprisingly, we find that all the models produce very similar results, almost independently of the adopted mass parameterization details, with only relatively larger variations in the cluster member component. The profiles of the extended cluster dark-matter halos are barely distinguishable, the different inner radial dependence of the PIEMD and PNFW mass models notwithstanding. This unexpected and interesting strong degeneracy can be ascribed to three effects: the measurement of the mass quantities (1) in projection, (2) within circular apertures, and (3) superposing two dark-matter halos in each lens model. We remark though that the differing central slope values of the PIEMD and PNFW mass density profiles are clearly visible in the reconstructed two-dimensional mass density maps, explaining partly the variance in the minimum$\chi^{2}$ values of Table 5. In summary, from these tests we can state that (1) the total mass measurements of a galaxy cluster from accurate strong lensing modeling are robust, even if different mass density profiles are adopted, (2) disparate mass density profiles produce detectable differences in the multiple image reconstruction, and (3) our values of the statistical and systematic uncertainties on the total mass of MACS 0416 are comparable and of the order of 5 per cent.

Finally, we estimate the profiles of the cumulative mass in the form of stars as a fraction of the cumulative total mass of MACS 0416, $M_{*} / M_{\mathrm{T}}(<R)$, as well as a fraction of the cluster member mass, $M_{*} / M_{\mathrm{G}}(<R)$. For a given radial (twodimensional) aperture, the stellar mass budget is obtained as the sum of the luminous mass values of the galaxy cluster members that have their luminosity centers enclosed within that aperture. The method used to measure the galaxy luminous mass values is described in Section 3.3.1 and the $M_{\mathrm{T}}(<R)$ and $M_{\mathrm{G}}(<R)$ profiles are those presented above and shown in Figure 15. We plot our results in Figure 18. We find that at projected distances from the cluster total mass center between 100 and $350 \mathrm{kpc}$ the stellar-to-total-cluster-mass ratio is slightly decreasing, with an average value of approximately $(1.0 \pm 0.3) \%$, and the stellar-to-total-galaxy-mass ratio has a fairly constant value of approximately $(7.7 \pm 3.6) \%$.

\section{COMPARISON WITH THE LITERATURE}

As mentioned in Section 1, MACS 0416 has been the subject of several recent strong lensing studies (Zitrin et al. 2013; Johnson et al. 2014; Richard et al. 2014; Jauzac et al. 2014, 2015; Diego et al. 2014). We discuss here the main differences and results of the previous analyses, in particular contrasting them with ours.

The total number of images of the modeled candidate strong lensing systems in MACS 0416 has more than doubled over the last year, increasing from 70 (Zitrin et al. 2013) to 194 (Jauzac et al. 2014). The combination of the shallow HST imaging from the CLASH survey with the more recent and deeper observations 

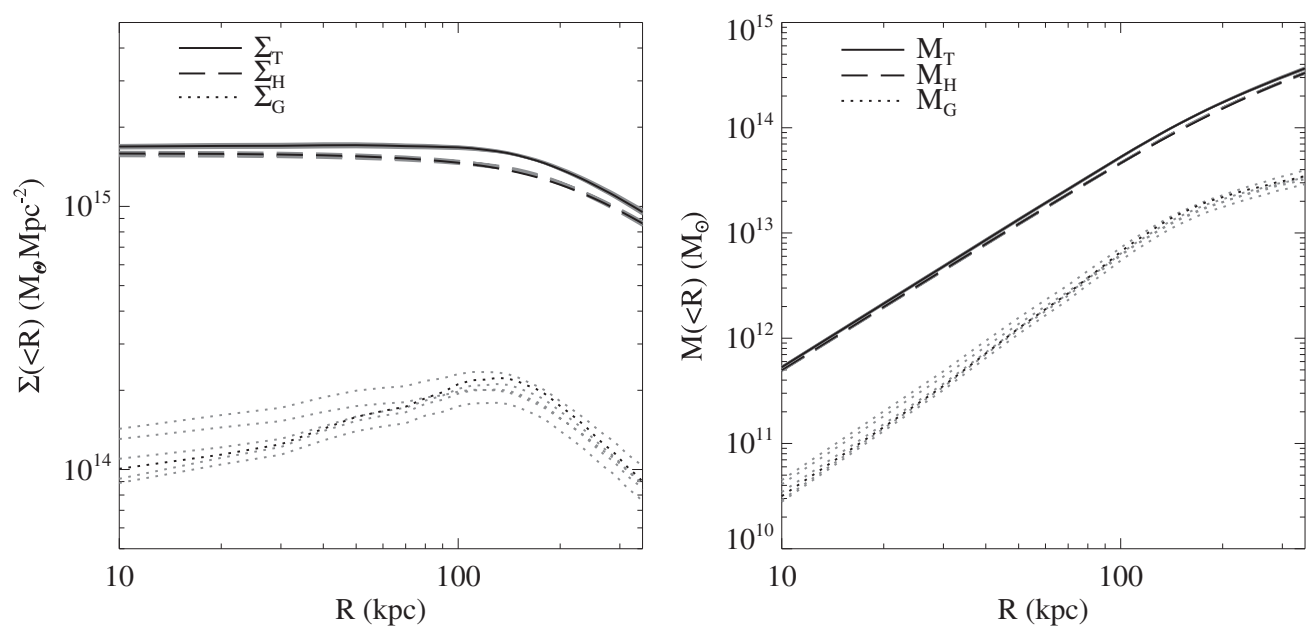

Figure 17. Surface mass density, $\Sigma(<R)$, and cumulative projected mass, $M(<R)$, profiles of the six optimized strong lensing models that include the 175 candidate cluster members (see Table 5). The 2PIEMD + 175(+1)dPIE $\left(M_{\mathrm{T}} L^{-1} \sim L^{0.2}\right)$ model is shown in black, all the others in gray. The solid, long-dashed, and dotted lines represent, respectively, the best-fitting total, cluster dark-matter halo, and cluster member profiles of the different models.

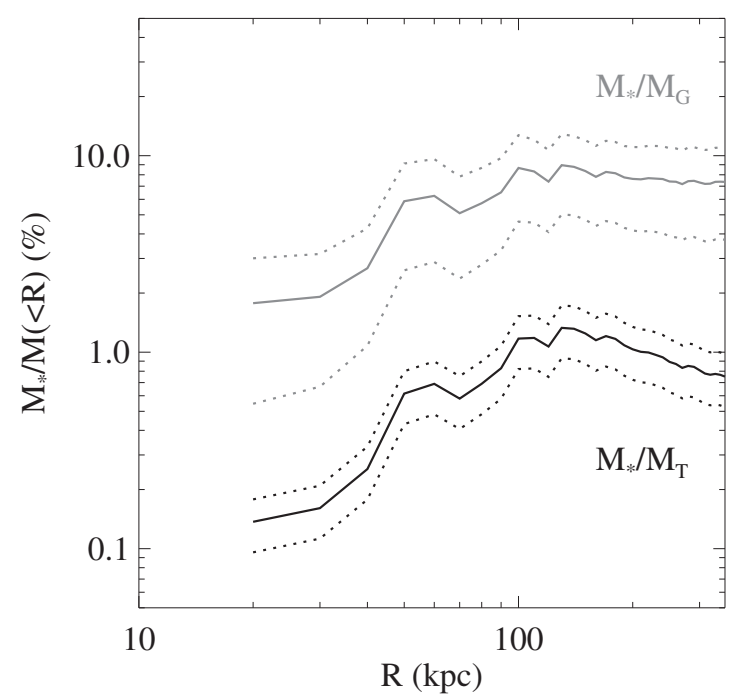

Figure 18. Cumulative projected profiles of the stellar over total cluster, $M_{*} / M_{\mathrm{T}}$, and stellar over total galaxy, $M_{*} / M_{\mathrm{G}}$, mass fractions. Solid and dotted lines show the median values and the $1 \sigma$ confidence levels, respectively.

in three HST/ACS filters from the HFF program has made such an improvement possible. More multiply imaged systems will likely be identified in the cluster core, thanks to the upcoming HFF data in the remaining four $H S T$ /WFC3 bands. The various aforementioned studies have considered different combinations of spectroscopic and photometric redshifts for the background lensed sources. The spectroscopic values adopted in all studies are those obtained from our CLASH-VLT survey and presented in Section 3.1. These measurements were shared with several international strong lensing groups to enable the distribution of preliminary strong lensing models (and magnification maps) to the community, before the acquisition of the HFF observations and the publication of this paper. The photometric redshifts were estimated from the multicolor HST photometry of CLASH (for more details, see Jouvel et al. 2014) and have been used as reference values or priors in the strong lensing modeling of previous studies. We restate here that we have purposely restricted our analysis to the positions, measured from the CLASH data, of the 30 multiple images associated with our first 10 spectroscopically confirmed strong lensing systems.
To reconstruct the total mass distribution of MACS 0416, most of the cited studies (Zitrin et al. 2013; Johnson et al. 2014; Jauzac et al. 2014; Diego et al. 2014) have focused on strong lensing only models, while some others have considered joint strong and weak lensing analyses (Richard et al. 2014; Jauzac et al. 2015). Various lensing codes (e.g., LTM, LENSTOOL, WSLAP+) have been used to assign mass, by means of physically motivated, parameterized profiles or pixelized grids, to the extended cluster dark-matter halos and candidate cluster members. The latter have essentially been selected from the cluster red sequence and their mass weights have been scaled according to their luminosity values in the reddest $H S T$ optical bands (i.e., the $\mathrm{F} 775 \mathrm{~W}$ or the $\mathrm{F} 814 \mathrm{~W}$ ). In our analysis, we have concentrated on the strong lensing modeling of MACS 0416 with GLEE (software) that makes use of parameterized mass profiles. We have determined the 175 candidate cluster members to include in our models by considering the full multicolor information, in 12 HST broadbands, of our more than 60 CLASH-VLT spectroscopically confirmed cluster members in the HST/WFC3 FoV. We have varied the cluster member mass contribution depending on the galaxy luminosity values in the reddest $H S T$ near-IR band (i.e., the F160W).

We confirm that the total mass distribution in the central regions of MACS 0416 is dominated by two highly elongated and close in projection components, representative of two massive and extended dark-matter halos and responsible for the large area on the lens plane with high magnification factors (as also observed in Figure 2 in Grillo \& Christensen 2011, on galaxy group scales). Consistent with previous results, we find that the inner mass density profile of the cluster is flat, with a core radius of the order of $100 \mathrm{kpc}$. The good agreement on these last points with the outcomes of the very different strong lensing models presented in Diego et al. (2014) is particularly remarkable. As far as aperture total mass measurements is concerned, we estimate that $(1) M_{\mathrm{T}}(<200 \mathrm{kpc})$ is between 1.72 and $1.77 \times 10^{14} M_{\odot}$, somewhat higher than the measurements of $(1.63 \pm 0.03) \times 10^{14} M_{\odot}$ and $(1.60 \pm 0.01) \times 10^{14} M_{\odot}$ presented in Richard et al. (2014) and Jauzac et al. (2014), respectively; (2) $M_{\mathrm{T}}(<250 \mathrm{kpc})$ ranges between 2.35 and $2.43 \times 10^{14} M_{\odot}$, consistent with the value of $2.46_{-0.08}^{+0.04} \times 10^{14} M_{\odot}$ reported in Johnson et al. (2014) (we also concur with the last authors in finding that the mass measurements of Zitrin et al. 2013 are noticeably higher than ours); (3) $M_{\mathrm{T}}(<320 \mathrm{kpc})$ is between 


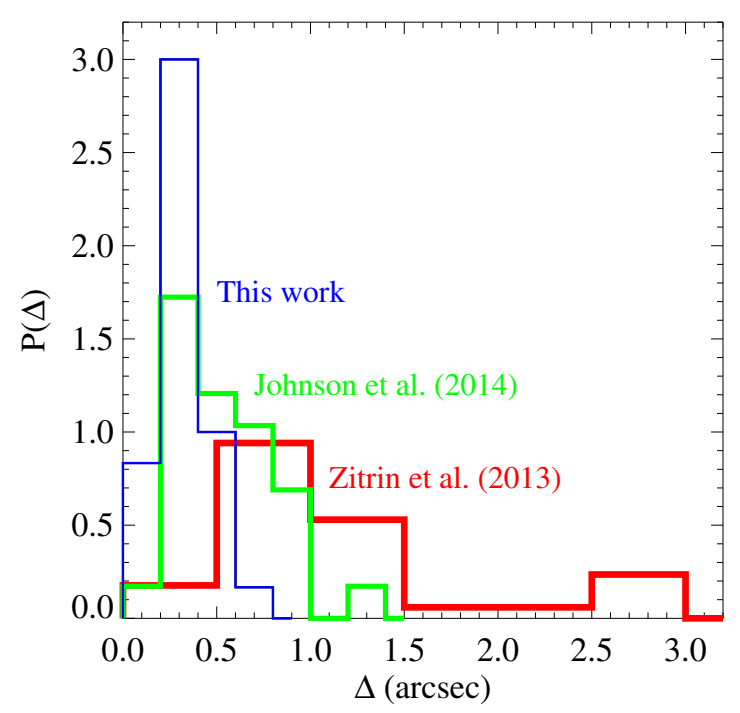

Figure 19. Probability distribution functions of the absolute values of the differences $(\Delta$, in arcsec) between the observed and best-fitting model-predicted positions of the multiple-image systems considered in the different strong lensing studies of MACS 0416.

3.23 and $3.35 \times 10^{14} M_{\odot}$, in agreement with the estimates of $(3.26 \pm 0.03) \times 10^{14} M_{\odot}$ and $(3.15 \pm 0.13) \times 10^{14} M_{\odot}$ from the strong and strong plus weak lensing analyses of, respectively, Jauzac et al. (2014) and Jauzac et al. (2015). The differences in the total mass measurements quoted here might partly be connected to the slight displacement in the adopted cluster mass centers, to the details of the lensing models, and, most likely, to the well-known (and, with photometric redshift values, only partially broken) degeneracy between the mass of a lens and the redshift of a multiply imaged source. We have explicitly checked that our total mass measurements do not depend appreciably on the redshift value of an individual multiple-image system, and, in particular, that the significant discrepancies listed above cannot be ascribed to the different redshift value of system 7 adopted in the previous analyses.

Despite the relatively good agreement obtained by the various groups on the cluster total mass reconstruction, we mention that the best-fitting values of some model parameters (see Table 7 in Section 3.5.2, Table 13 in Johnson et al. 2014, Table 8 in Richard et al. 2014, and Table 1 in Jauzac et al. 2014) are significantly inconsistent. These discrepancies are likely due to the use of different multiple image systems as constraints, modeling assumptions and strategies. Rather than discussing in length on the intricate modeling details, we focus instead on the goodness of the different models in reproducing the positions of the observed multiple images, which can be compared irrespectively of model assumptions. To quantify the goodness of fit, we consider the values of $\Delta$, the modulus of the difference (in arcsec) between the observed and bestfitting model-predicted positions of an image. In Figure 19, we compare the probability distribution functions of $\Delta$ estimated from our best-fitting, minimum- $\chi^{2}$ strong lensing model and those of Zitrin et al. (2013) and Johnson et al. (2014) (the only two previous studies that present the numbers necessary for this comparison). We note that for the distribution of $\Delta$ for Johnson et al. (2014) we consider exactly our 10 multiple image systems, whereas for Zitrin et al. (2013) we use the positions of 34 multiple images from 13 different sources (23 images of 8 sources are in common with ours). From Figure 19, it is evident that our model reproduces the observables with extremely good accuracy. More quantitatively, the median values of $\Delta$ are 0.31 in our case, 0.44 in Johnson et al. (2014) and 0'.90 in Zitrin et al. (2013). Specifically, the rms $\Delta$ is 0.36 in our study, $0^{\prime \prime} .51$ in Johnson et al. (2014), 0'.'68 in Jauzac et al. (2014), approximately 0 . 8 in Richard et al. (2014), and 1".37 in Zitrin et al. (2013). ${ }^{17}$

From the discussion above, we caution that strong lensing models that reproduce the positions of observed multiple images with an accuracy worse than ours should hardly enable total mass measurements of MACS 0416 with a precision better than ours (approximately 5\% in both statistical and systematical uncertainties). Moreover, we remark that the knowledge of the spectroscopic redshifts of several cluster members and multiply imaged sources are essential to build detailed and reliable strong lensing models, and thus to obtain accurate total mass estimates of a galaxy cluster. The spectra collected within our CLASHVLT survey will also allow us to detect the presence of possible mass structures along the line of sight, usually not accounted for in the strong lensing modeling, and to quantify their effects on the offset between the observed and model-predicted image positions. Our values of $\Delta$ of less than 0.4 suggests that the compound lensing effect is weaker than previously thought and usually assumed until now (approximately 1"; e.g., Host 2012).

\section{COMPARISON WITH COSMOLOGICAL SIMULATIONS}

The high-quality mass reconstruction of MACS 0416, enabled by the combination of superb HST imaging and extensive VLT spectroscopy, allows a detailed investigation of the inner structure of the dark-matter mass distribution in this system and a meaningful comparison with cosmological simulations. We juxtapose the measured cluster mass profile and the amount of substructures (cluster galaxy members) identified in MACS 0416 with theoretical predictions based on a set of $N$-body simulations in order to probe the formation history of the galaxy cluster and its substructures. In Section 5.1, we outline the $\mathrm{N}$-body simulations. The comparison of the cluster mass profile is presented in Section 5.2 whereas the comparison of substructure properties is in Section 5.3. An extension of this kind of analyses to a sample of galaxy clusters, presenting different properties in terms of total mass and dynamical state, will be possible through accurate modeling of further CLASH-VLT targets.

\subsection{High-resolution Simulation of Galaxy Clusters}

The set of $N$-body simulations consists of 29 Lagrangian regions, extracted around massive clusters from a cosmological box, and resimulated at high resolution using the "zoom-in" technique (Tormen et al. 1997). We refer the reader to Bonafede et al. (2011) and Contini et al. (2012) for a detailed description of this set of simulations. The adopted cosmological model has $\Omega_{\mathrm{m}}=0.24$ for the matter density parameter, $\Omega_{\mathrm{bar}}=0.04$ for the contribution of baryons, $H_{0}=72 \mathrm{~km} \mathrm{~s}^{-1} \mathrm{Mpc}^{-1}$ for the present-day Hubble constant, $n_{s}=0.96$ for the primordial spectral index, and $\sigma_{8}=0.8$ for the normalization of the power spectrum.

The particle mass is $10^{8} M_{\odot} h^{-1}$, with a Plummer-equivalent softening length for the computation of the gravitational force fixed to $\epsilon=2.3 h^{-1} \mathrm{kpc}$ in physical units at redshift $z<2$, and in comoving units at higher redshift. Each output of the 17 We note though that different analyses use different sets of multiple image
systems. 


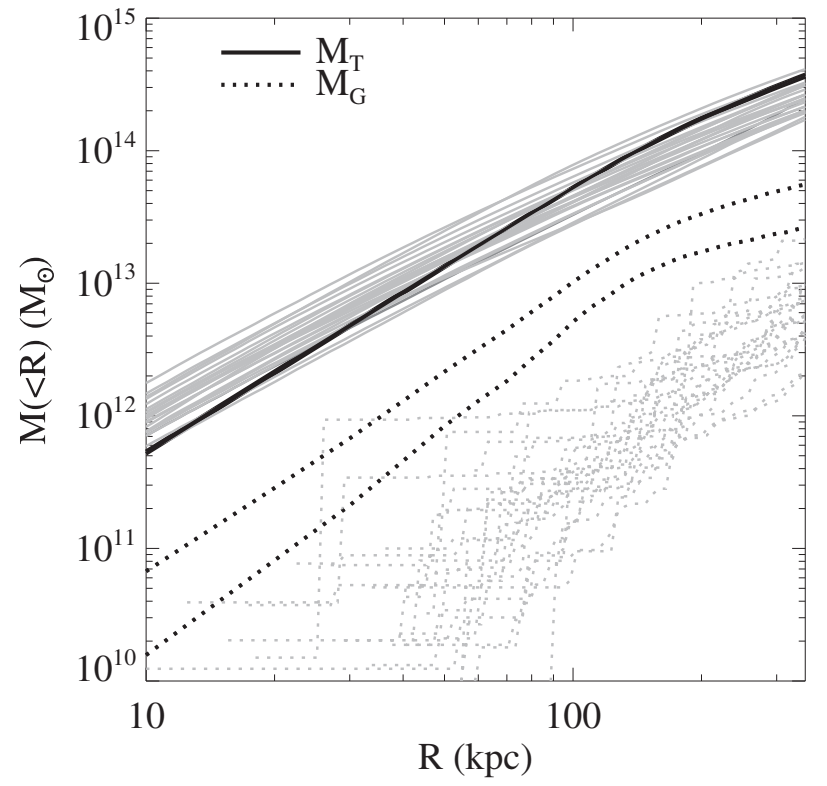

Figure 20. Cumulative projected mass, $M(<R)$, profiles from the best-fitting strong lensing model of MACS 0416 (in black) and from dark-matter-only cosmological simulations of galaxy clusters (in gray). The solid and dotted lines represent, respectively, the total and cluster member profiles, at $1 \sigma$ confidence level for MACS 0416 and as different clusters for the simulations.

simulation (93 in total between $z \sim 60$ and $z=0$ ) has been postprocessed to identify dark matter halos and subhalos. Dark matter halos have been identified using a standard friends-offriends (FOF) algorithm, with a linking length of 0.16 in units of the mean inter-particle separation. Each FOF group was then decomposed into a set of disjoint subhalos using the algorithm SUBFIND (Springel et al. 2001).

As in previous work, we consider as genuine subhalos only those that retain at least 20 bound particles after a gravitational unbinding procedure. Our simulation thus contain all subhalos that are more massive than $2 \times 10^{9} M_{\odot} h^{-1}$ (corresponding to the 20 particle limit). We further impose that these subhalos have circular velocities greater than $50 \mathrm{~km} \mathrm{~s}^{-1}$. The circular velocity is computed as the maximum value of $\sqrt{G M(<r) / r}$ where $M(<r)$ is the mass within a distance $r$ from the center of the subhalos. These limits on mass and circular velocity are meant to select the largest number of well defined subhalos. We have checked that for the adopted velocity threshold we obtain a tight relation between the mass and the velocity dispersion of subhalos, making the circular velocity a good proxy for subhalo mass.

\subsection{Cluster Mass Profile}

To compare with the measured cumulative projected mass profile of MACS 0416 in Section 3.5, we consider all the simulated halos above $5 \times 10^{14} M_{\odot}$ at a redshift of 0.46 , the closest redshift to that of MACS 0416 , for a total of 24 systems. For each halo we choose a random preferential axis and compute the total mass within projected (two-dimensional) radii, as shown in Figure 20. We also show the mass profile associated with the identified subhalos with masses $>2 \times 10^{9} M_{\odot} h^{-1}$ and circular velocities $>50 \mathrm{~km} \mathrm{~s}^{-1}$. For the galaxy members in MACS 0416, we also include only those (165 out of 175) that have circular velocities ${ }^{18}>50 \mathrm{~km} \mathrm{~s}^{-1}$.

\footnotetext{
18 The circular velocities of the cluster galaxies can be computed through the
} resulting $\vartheta_{\mathrm{E}, i}$ of each galaxy, Equations (7) and (8).

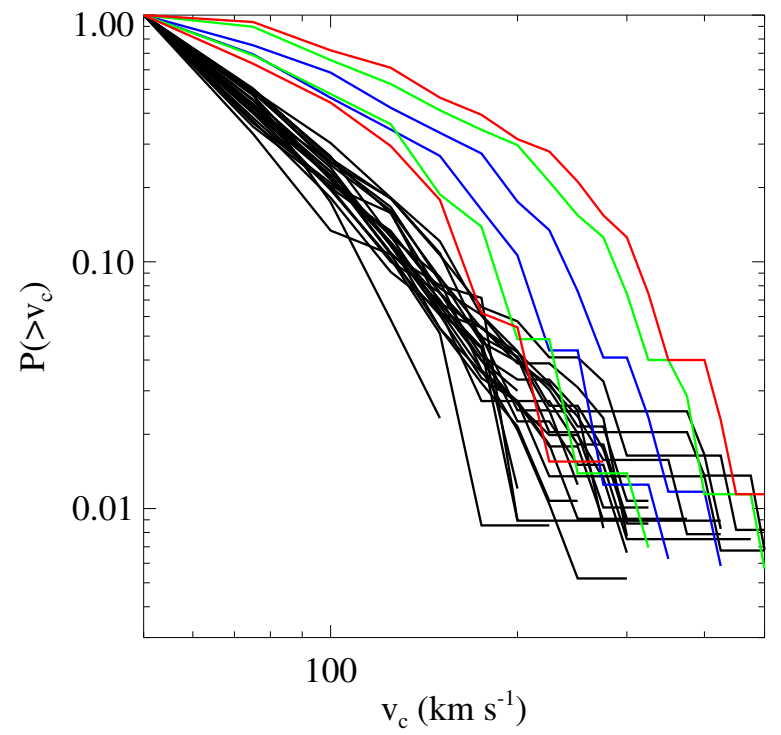

Figure 21. Subhalo velocity functions, i.e., probability distribution functions of subhalos with circular velocities larger than fixed values, of massive galaxy clusters estimated from cosmological simulations and our strong lensing modeling. The simulated (in black) and observed (in color) functions, measured within a two-dimensional aperture of $420 \mathrm{kpc}$, are normalized to their corresponding values at $50 \mathrm{~km} \mathrm{~s}^{-1}$. The $1 \sigma, 2 \sigma$, and $3 \sigma$ confidence levels, according to the best-fitting 2PIEMD $+175(+1)$ dPIE $\left(M_{\mathrm{T}} L^{-1} \sim L^{0.2}\right)$ model of MACS 0416, are shown in blue, green, and red, respectively.

Comparing to the simulated $M(<R)$ in gray in Figure 20, the observed cumulative projected mass profile of MACS 0416 (black curve) traces the range spanned by the simulated curves given our selection of simulated halos with masses similar to MACS 0416. In detail, the observed $M_{\mathrm{T}}(<R)$ increases more quickly as a function of radius away from the cluster center, implying that the observed total mass of the cluster MACS 0416 has a shallower projected core density profile than the simulated ones. This could perhaps be explained by the apparent merging state of MACS 0416 in the plane of the sky, although we note that several of the simulated clusters are in similar dynamical states.

Interestingly, despite the similar total masses of MACS 0416 and of the simulated clusters, the cumulative projected mass of the cluster galaxies, $M_{\mathrm{G}}(<R)$, in MACS 0416 is in general larger than that of the subhalos in the simulated clusters, especially at radius $R \gtrsim 60 \mathrm{kpc}$. This offset in mass could be due to smaller masses of subhalos, or fewer numbers of subhalos in the simulations, and we explore this difference in more detail next.

\subsection{Distribution of Substructures}

In Figure 21, we plot the velocity function of the cluster galaxies in MACS 0416 (color curves) and of the subhalos in the simulated clusters (black curves) within $420 \mathrm{kpc}$ (corresponding approximately to the $H S T / \mathrm{WFC} 3 \mathrm{FoV}$ at $z=0.396$ ). As before, these substructures have circular velocities larger than $50 \mathrm{~km} \mathrm{~s}^{-1}$. Overall, the observed velocity function is higher and has a different shape from the power-law like velocity function from simulations.

To probe further the discrepancy between observations and simulations, we plot the numbers of cluster galaxies and simulated subhalos in Figure 22. In both panels, the histograms show the distributions of the observed number of cluster 

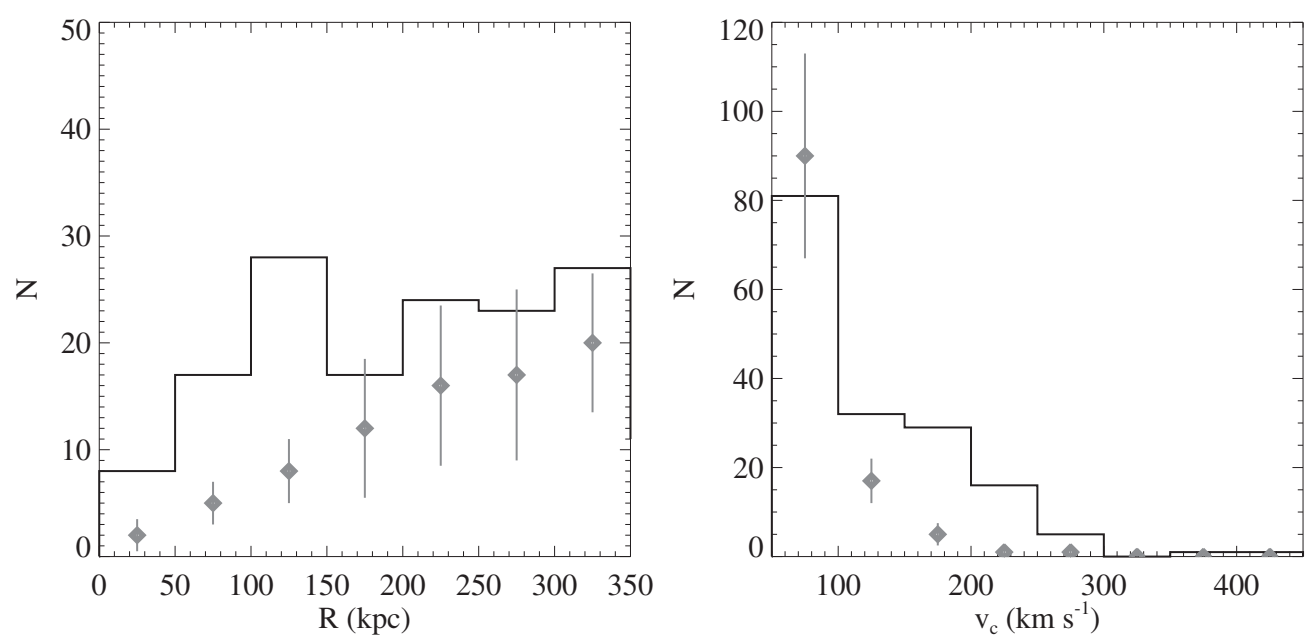

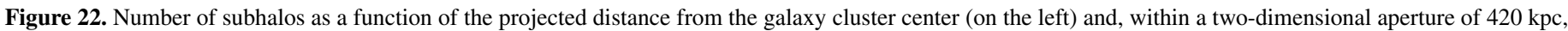

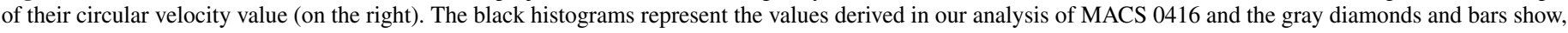
correspondingly, the median values and the $1 \sigma$ uncertainties obtained from cosmological simulations.

galaxies in MACS 0416, whereas the diamond points are the medians with $1 \sigma$ uncertainties from the simulated clusters. The simulated halos consistently underpredict the number of subhalos on all radial scales, as shown in the left panel. The underprediction is more severe in the inner $\sim 150 \mathrm{kpc}$ of galaxy clusters. In the right panel, we categorize the substructures in terms of their circular velocities (i.e., masses) regardless of their locations within the clusters. We find that the observed number of low-mass cluster members with circular velocities $\lesssim 100 \mathrm{~km} \mathrm{~s}^{-1}$ is in good agreement with the predicted number from the $N$-body simulations, whereas the simulated clusters have fewer substructures with circular velocities between $\sim 100 \mathrm{~km} \mathrm{~s}^{-1}$ and $\sim 300 \mathrm{~km} \mathrm{~s}^{-1}$. We remark that within this last circular velocity range the results of our observations are robust, since our sample of candidate cluster members can only be marginally contaminated by foreground/background objects at the corresponding near-IR luminosities (see Section 3.3.1). In fact, we have checked that by varying from 0.5 to 0.9 the value of the probability threshold of our method used to obtain the cluster members for the strong lensing analysis, i.e., moving from a more complete to a purer sample of cluster members, the number of selected bright (and massive) galaxies, with $F 160 W<21$ mag (i.e., stellar mass values larger than $10^{9.8} M_{\odot}$ ), changes from 73 to 69 . This confirms that the number of candidate bright cluster members, and thus the comparison with cosmological simulations at the corresponding circular velocities, does not depend appreciably on the selection details.

These findings suggest that the massive subhalos are not formed or accreted into the simulated clusters as quickly as observed, or that tidal strippings of massive subhalos are more efficient than observed, or a combination of these effects. Also, it appears that the tidal disruptions of galaxies in the inner parts of MACS 0416 might be less than those in the simulations, given the higher number of cluster galaxies in MACS 0416. We note that the simulations do not contain baryons. The addition of baryons into subhalos would likely make the subhalos more tightly bound, which would in turn make tidal stripping less effective and result in a higher number of subhalos from simulations. This could perhaps partly explain the lower number of subhalos in simulations, although the effect might not affect significantly the number of massive subhalos. We defer the comparison of substructure distributions in hydro simulations to future work which will provide insights on the formation of galaxy clusters and the role of baryons.

\section{SUMMARY}

Thanks to the excellent HST panchromatic observations and VLT spectra of multiply imaged sources and galaxy cluster members from our CLASH and CLASH-VLT programs, we have performed a thorough strong lensing study and comparison with dark-matter only cosmological simulations in the inner regions of the galaxy cluster MACS 0416.

We have emphasized that the use of only multiple-image systems with spectroscopic redshifts and of a pure sample of cluster members, selected through extensive multi-color and spectroscopic information, is key to constructing robust mass maps with high angular resolution. Further insights into the small scale structure of the cluster dark-matter distribution would require the measurement of the cluster member internal velocity dispersions, which we are pursuing. The main results of our analysis can be summarized as follows:

1. We reconstruct the observed image positions of 30 multiple images from 10 different sources, with spectroscopic redshift values between 1.637 and 3.223, with an unprecedented accuracy of approximately 0.3 .

2. The mass model that best fits the lensing observables is constituted of two cored elliptical pseudo-isothermal components and 175 dual elliptical pseudo-isothermal components, representing the extended cluster dark-matter halos and candidate cluster members, respectively. The latter have been selected by using the full covariance matrix of the color distribution of the spectroscopic members.

3. The two cluster dark-matter halos have mass centers at significant projected distances from the luminosity centers of the two brightest cluster galaxies and core radii larger than $50 \mathrm{kpc}$.

4. When estimated within circular apertures, the total surface mass density shows a flat inner profile out to more than $100 \mathrm{kpc}$. The cumulative projected total mass is accurate to $5 \%$ (of both statistical and systematical uncertainties) out to $350 \mathrm{kpc}$, where it notably matches independent weak lensing measurements. 
5. The galaxy cluster members are best modeled by mass profiles that have total mass-to-light ratios increasing with the galaxy near-IR (F160W) luminosities. At more than $100 \mathrm{kpc}$ in projection from the cluster barycenter, the mass in the form of stars represents approximately $8 \%$ of the cluster members' total mass and $1 \%$ of the cluster total mass.

6. We find that simulated galaxy clusters with total mass values comparable to that of MACS 0416 contain considerably less mass in subhalos in their cores relative to MACS 0416. The mismatch is more evident within the central $150 \mathrm{kpc}$ and is associated with a deficiency in massive substructures with circular velocities $\gtrsim 100 \mathrm{~km} \mathrm{~s}^{-1}$.

The new high level of accuracy we have reached in reproducing the observed multiple image positions of spectroscopically confirmed sources paves the way for effectively studying the perturbing lensing effect of mass structures along the line of sight. It also lays the groundwork for measuring the values of cosmological parameters via ratios of angular diameter distances of the multiple background sources at different redshifts from a sample of massive strong lensing clusters. Moreover, our findings in MACS 0416 of the cored inner density profiles of the two cluster dark-matter halos and their significant offsets from the brightest cluster galaxies, together with forthcoming deep Chandra observations, might reveal important clues about the nature of dark matter (e.g., self-interacting or not). Further investigations following our pilot juxtaposition of precise observational results and predictions from $N$-body simulations in the mass structure of galaxy clusters will enable tests of the very physical foundations of the current $\Lambda C D M$ model and the impact of baryonic physics on the mass assembly of cosmological structures. The exceptional CLASH/HFF imaging data and the spectroscopic follow-up from the ground like CLASH-VLT, will be key to achieving all these aims.

We thank the ESO User Support group, and specifically Vincenzo Mainieri, for the continuous and excellent support on the implementation of the Large Programme 186.A-0798. The CLASH Multi-Cycle Treasury Program is based on observations made with the NASA/ESA Hubble Space Telescope. The Space Telescope Science Institute is operated by the Association of Universities for Research in Astronomy, Inc., under NASA contract NAS 5-26555. ACS was developed under NASA Contract NAS 5-32864. This research is supported in part by NASA Grant HST-GO-12065.01-A. The Dark Cosmology Centre is funded by the DNRF. We acknowledge partial support by the DFG Cluster of Excellence Origin Structure of the Universe. G.B.C. is supported by the CAPES-ICRANET program through the grant BEX 13946/13-7. We acknowledge financial support from MIUR PRIN2010-2011 (J91J12000450001). K.U. acknowledges support from the National Science Council of Taiwan (grant NSC100-2112-M-001-008-MY3). Support for A.Z. is provided by NASA through Hubble Fellowship grant HST-HF-51334.01-A awarded by STScI. V.P. acknowledges a grant from "Consorzio per la Fisica - Trieste." A.F. acknowledges the support by INAF PRIN 2010 grant (VIPERS).

\section{REFERENCES}

Bender, R., Burstein, D., \& Faber, S. M. 1992, ApJ, 399, 462

Biviano, A., Rosati, P., Balestra, I., et al. 2013, A\&A, 558, 1

Bonafede, A., Dolag, K., Stasyszyn, F., Murante, G., \& Borgani, S. 2011, MNRAS, 418, 2234

Bruzual, G., \& Charlot, S. 2003, MNRAS, 344, 1000

Calzetti, D., Armus, L., Bohlin, R. C., et al. 2000, ApJ, 533, 682

Coe, D., Umetsu, K., Zitrin, A., et al. 2012, ApJ, 757, 22

Contini, E., De Lucia, G., \& Borgani, S. 2012, MNRAS, 420, 2978

De Lucia, G., Kauffmann, G., Springel, V., et al. 2004, MNRAS, 348, 333

Diego, J. M., Broadhurst, T., Molnar, S. M., Lam, D., \& Lim, J. 2014, arXiv: 1406.1217

Donahue, M., Voit, G. M., Mahdavi, A., et al. 2014, ApJ, 794, 136

Dunkley, J., Bucher, M., Ferreira, P. G., Moodley, K., \& Skordis, C. 2005, MNRAS, 356, 925

Eichner, T., Seitz, S., Suyu, S. H., et al. 2013, ApJ, 774, 124

Elíasdóttir, Á., Limousin, M., Richard, J., et al. 2007, arXiv:0710.5636

Faber, S. M., Dressler, A., Davies, R. L., Burstein, D., \& Lynden-Bell, D. 1987, in Nearly Normal Galaxies, ed. S. M. Faber (New York: Springer), 175

Gao, L., White, S. D. M., Jenkins, A., Stoehr, F., \& Springel, V. 2004, MNRAS, 355,819

Ghigna, S., Moore, B., Governato, F., et al. 1998, MNRAS, 300, 146

Grillo, C., \& Christensen, L. 2011, MNRAS, 418, 929

Grillo, C., Gobat, R., Lombardi, M., \& Rosati, P. 2009, A\&A, 501, 461

Grillo, C., Gobat, R., Presotto, V., et al. 2014, ApJ, 786, 11

Host, O. 2012, MNRAS, 420, 18

Jauzac, M., Clément, B., Limousin, M., et al. 2014, MNRAS, 443, 1549

Jauzac, M., Jullo, E., Eckert, D., et al. 2015, MNRAS, 446, 4132

Johnson, T. L., Sharon, K., Bayliss, M. B., et al. 2014, ApJ, 797, 48

Jouvel, S., Host, O., Lahav, O., et al. 2014, A\&A, 562, 86

Kassiola, A., \& Kovner, I. 1993, ApJ, 417, 450

Klypin, A., Kravtsov, A. V., Valenzuela, O., \& Prada, F. 1999, ApJ, 522,82

Koekemoer, A. M., Aussel, H., Calzetti, D., et al. 2007, ApJS, 172, 196

Koekemoer, A. M., Faber, S. M., Ferguson, H. C., et al. 2011, ApJS, 197, 36

Le Fèvre, O., Saisse, M., Mancini, D., et al. 2003, Proc. SPIE, 4841, 1670

Lemze, D., Postman, M., Genel, S., et al. 2013, ApJ, 776, 91

Mann, A. W., \& Ebeling, H. 2012, MNRAS, 420, 2120

Medezinski, E., Umetsu, K., Nonino, M., et al. 2013, ApJ, 777, 43

Meneghetti, M., Rasia, E., Vega, J., et al. 2014, ApJ, 797, 34

Merritt, D. 1983, ApJ, 264, 24

Merten, J., Meneghetti, M., Postman, M., et al. 2014, arXiv:1404.1376

Moore, B., Ghigna, S., Governato, F., et al. 1999, ApJ, 524, 19

Natarajan, P., De Lucia, G., \& Springel, V. 2007, MNRAS, 376, 180

Natarajan, P., Kneib, J.-P., Smail, I., et al. 2009, ApJ, 693, 970

Navarro, J. F., Frenk, C. S., \& White, S. D. M. 1996, ApJ, 462, 563

Navarro, J. F., Frenk, C. S., \& White, S. D. M. 1997, ApJ, 490, 493

Oguri, M., Lee, J., \& Suto, Y. 2003, ApJ, 599, 7

Postman, M., Coe, D., Benítez, N., et al. 2012, ApJS, 199, 25

Richard, J., Jauzac, M., Limousin, M., et al. 2014, MNRAS, 444, 268

Rousseeuw, P. J. 1984, J. Am. Stat. Assoc., 79, 871

Salpeter, E. E. 1955, ApJ, 121, 161

Springel, V., White, S. D. M., Tormen, G., \& Kauffmann, G. 2001, MNRAS, 328,726

Suyu, S. H., \& Halkola, A. 2010, A\&A, 524, A94

Suyu, S. H., Hensel, S. W., McKean, J. P., et al. 2012, ApJ, 750, 10

Tormen, G. 1997, MNRAS, 290, 411

Tormen, G., Bouchet, F. R., \& White, S. D. M. 1997, MNRAS, 286, 865

Tormen, G., Diaferio, A., \& Syer, D. 1998, MNRAS, 299, 728

Umetsu, K., Broadhurst, T., Zitrin, A., Medezinski, E., \& Hsu, L.-Y. 2011, ApJ, 729,127

Umetsu, K., Medezinski, E., Nonino, M., et al. 2012, ApJ, 755, 56

Umetsu, K., Medezinski, E., Nonino, M., et al. 2014, ApJ, 795, 163

Wechsler, R. H., Bullock, J. S., Primack, J. R., Kravtsov, A. V., \& Dekel, A. 2002, ApJ, 568, 52

Zhao, D. H., Mo, H. J., Jing, Y. P., \& Börner, G. 2003, MNRAS, 339, 12

Zitrin, A., Meneghetti, M., Umetsu, K., et al. 2013, ApJ, 762, 30 